\title{
Convergence to the Product of the Standard Spheres and Eigenvalues of the Laplacian
}

\author{
Masayuki AINO
}

RIKEN, Center for Advanced Intelligence Project AIP, 1-4-1 Nihonbashi, Tokyo 103-0027, Japan

E-mail: masayuki.aino@riken.jp

URL: https://sites.google.com/site/masayukiaino/

Received July 17, 2020, in final form February 07, 2021; Published online February 24, 2021 https://doi.org/10.3842/SIGMA.2021.017

\begin{abstract}
We show a Gromov-Hausdorff approximation to the product of the standard spheres $S^{n-p} \times S^{p}$ for Riemannian manifolds with positive Ricci curvature under some pinching condition on the eigenvalues of the Laplacian acting on functions and forms.
\end{abstract}

Key words: Gromov-Hausdorff distance; Lichnerowicz-Obata estimate; parallel $p$-form 2020 Mathematics Subject Classification: 53C20; 58J50

\section{Introduction}

In this article we show that if an $n$-dimensional closed Riemannian manifold with positive Ricci curvature admits an almost parallel $p$-form $(2 \leq p<n / 2)$ in $L^{2}$-sense and if the first $n+1$ eigenvalues of the Laplacian acting on functions are close to their optimal values, then the Riemannian manifold is close to the product of the standard spheres $S^{n-p} \times S^{p}$ with appropriate radii (Main Theorem below). Before giving the precise statement, we provide some backgrounds.

The Lichnerowicz-Obata theorem is one of the classical theorem about the first eigenvalue of the Laplacian. Lichnerowicz showed the optimal comparison result for the first eigenvalue when the Riemannian manifold has positive Ricci curvature, and Obata showed that the equality of the Lichnerowicz estimate implies that the Riemannian manifold is isometric to the standard sphere. In the following, $\lambda_{k}(g)$ denotes the $k$-th positive eigenvalue of the minus Laplacian $-\Delta:=-\operatorname{tr}_{g}$ Hess acting on functions.

Theorem 1.1 (Lichnerowicz-Obata theorem). Take an integer $n \geq 2$. Let $(M, g)$ be an $n$ dimensional closed Riemannian manifold. If Ric $\geq(n-1) g$, then $\lambda_{1}(g) \geq n$. The equality $\lambda_{1}(g)=n$ holds if and only if $(M, g)$ is isometric to the standard sphere of radius 1 .

Petersen [26], Aubry [8] and Honda [21] showed the stability result of the LichnerowiczObata theorem. In the following, $d_{\mathrm{GH}}$ denotes the Gromov-Hausdorff distance and $S^{n}$ denotes the $n$-dimensional standard sphere of radius 1 (see Definition 2.13 for the definition of the Gromov-Hausdorff distance).

Theorem $1.2([8,21,26])$. For given an integer $n \geq 2$ and a positive real number $\epsilon>0$, there exists $\delta(n, \epsilon)>0$ such that if $(M, g)$ is an $n$-dimensional closed Riemannian manifold with Ric $\geq(n-1) g$ and $\lambda_{n}(g) \leq n+\delta$, then $d_{\mathrm{GH}}\left(M, S^{n}(1)\right) \leq \epsilon$.

Note that Petersen considered the pinching condition on $\lambda_{n+1}(g)$, and Aubry and Honda improved it independently.

This paper is a contribution to the Special Issue on Scalar and Ricci Curvature in honor of Misha Gromov on his 75th Birthday. The full collection is available at https://www.emis.de/journals/SIGMA/Gromov.html 
When the Riemannian manifold admits a non-trivial parallel differential form, we have the stronger estimate.

Theorem 1.3 ([1, 18]). Let $(M, g)$ be an $n$-dimensional closed Riemannian manifold. Assume that Ric $\geq(n-p-1) g$ and that there exists a nontrivial parallel $p$-form on $M(2 \leq p \leq n / 2)$. Then, we have $\lambda_{1}(g) \geq n-p$. Moreover, if $p<n / 2$ and $\lambda_{n-p+1}(g)=n-p$ hold, then $(M, g)$ is isometric to a product $S^{n-p}(1) \times\left(X, g^{\prime}\right)$, where $\left(X, g^{\prime}\right)$ is some $p$-dimensional closed Riemannian manifold.

To simplify the numbers appearing in the theorem, we consider the assumption Ric $\geq$ $(n-p-1) g$ instead of Ric $\geq(n-1) g$. By scaling, the estimate in Theorem 1.1 becomes $\lambda_{1}(g) \geq n(n-p-1) /(n-1)$ when Ric $\geq(n-p-1) g$. Note that we have $n-p>n(n-p-1) /(n-1)$.

To state the almost version of Theorem 1.3, we introduce the first eigenvalue of the connection Laplacian acting on $p$-forms $\lambda_{1}\left(\Delta_{C, p}\right)$ for a closed Riemannian manifold $(M, g)$ :

$$
\lambda_{1}\left(\Delta_{C, p}\right):=\inf \left\{\frac{\|\nabla \omega\|_{L^{2}}^{2}}{\|\omega\|_{L^{2}}^{2}}: \omega \in \Gamma\left(\bigwedge^{p} T^{*} M\right) \text { with } \omega \neq 0\right\} .
$$

Note that there exists a non zero $p$-form $\omega$ with $\|\nabla \omega\|_{L^{2}}^{2} \leq \delta\|\omega\|_{L^{2}}^{2}$ for some $\delta>0$ if and only if $\lambda_{1}\left(\Delta_{C, p}\right) \leq \delta$ holds. For arbitrary integers $n, p$ with $2 \leq p \leq n / 2$ and a real number $\epsilon>0$, considering a small perturbation of $S^{n-p}(1) \times S^{p}\left(r_{n, p}\right)$, we can find an $n$-dimensional closed Riemannian manifold with Ric $\geq(n-p-1) g$ such that $0<\lambda_{1}\left(\Delta_{C, p}\right)<\epsilon$ holds. Here we defined $r_{n, p}:=\sqrt{(p-1) /(n-p-1)}$. In other words, we do not have the gap theorem for the first eigenvalue of the connection Laplacian $\lambda_{1}\left(\Delta_{C, p}\right)$ if we only assume a lower Ricci curvature bound.

Let us state the almost version of the eigenvalue estimate.

Theorem 1.4 ([1]). For given integers $n \geq 4$ and $2 \leq p \leq n / 2$, there exists a constant $C(n, p)>0$ such that if $(M, g)$ is an $n$-dimensional closed Riemannian manifold with $\mathrm{Ric}_{g} \geq$ $(n-p-1) g$, then we have

$$
\lambda_{1}(g) \geq n-p-C(n, p) \lambda_{1}\left(\Delta_{C, p}\right)^{1 / 2} .
$$

This theorem recovers the estimate in Theorem 1.3 when $\lambda_{1}\left(\Delta_{C, p}\right)=0$.

We next state the approximation result to the product space.

Theorem 1.5 ([1]). For given integers $n \geq 5$ and $2 \leq p<n / 2$ and a positive real number $\epsilon>0$, there exists $\delta=\delta(n, p, \epsilon)>0$ such that if $(M, g)$ is an $n$-dimensional closed Riemannian manifold with $\operatorname{Ric}_{g} \geq(n-p-1) g$,

$$
\lambda_{n-p+1}(g) \leq n-p+\delta
$$

and

$$
\lambda_{1}\left(\Delta_{C, p}\right) \leq \delta,
$$

then $M$ is orientable and

$$
d_{\mathrm{GH}}\left(M, S^{n-p}(1) \times X\right) \leq \epsilon,
$$

where $X$ is some compact metric space. 
In this article we study the structure of the metric space $X$ in this theorem and show that $X$ with some appropriate Borel measure satisfies the $\operatorname{RCD}^{*}(n-p-1, p)$ condition (see Proposition 3.2), which means a synthetic notion of "Ric $\geq n-p-1$ and $\operatorname{dim} \leq p$ with Riemannian structure" (see Definition 2.6). As a consequence, we can show the estimate $\lambda_{n-p+2}(g) \geq p(n-p-1) /(p-1)-\epsilon$ under the assumption of Theorem 1.5 (see Theorem 4.1) and the following theorem.

Main Theorem. For given integers $n \geq 5$ and $2 \leq p<n / 2$ and a positive real number $\epsilon>0$, there exists $\delta=\delta(n, p, \epsilon)>0$ such that if $(M, g)$ is an $n$-dimensional closed Riemannian manifold with $\operatorname{Ric}_{g} \geq(n-p-1) g$,

$$
\lambda_{n-p+1}(g) \leq n-p+\delta, \quad \lambda_{n+1}(g) \leq \frac{p(n-p-1)}{p-1}+\delta
$$

and

$$
\lambda_{1}\left(\Delta_{C, p}\right) \leq \delta
$$

then

$$
d_{\mathrm{GH}}\left(M, S^{n-p}(1) \times S^{p}\left(\sqrt{\frac{p-1}{n-p-1}}\right)\right) \leq \epsilon,
$$

and $M$ is diffeomorphic to $S^{n-p} \times S^{p}$.

We have the last assertion by the topological stability theorem due to Cheeger-Colding [12, Theorem A.1.12]. We show the main theorem including the case when $\lambda_{1}\left(\Delta_{C, n-p}\right) \leq \delta$ (see Theorem 4.2).

\section{Preliminaries}

\subsection{Basic notation}

We first recall some basic definitions and fix our convention.

Let $(M, g)$ be a closed Riemannian manifold. For any $p \geq 1$, we use the normalized $L^{p}$-norm:

$$
\|f\|_{L^{p}}^{p}:=\frac{1}{\operatorname{Vol}(M)} \int_{M}|f|^{p} \mathrm{~d} \mu_{g}
$$

and $\|f\|_{L^{\infty}}:=\underset{x \in M}{\operatorname{ess} \sup }|f(x)|$ for a measurable function $f$ on $M$. We also use these notation for tensors. We have $\|f\|_{L^{p}} \leq\|f\|_{L^{q}}$ for any $p \leq q \leq \infty$.

Let $\nabla$ denote the Levi-Civita connection. Throughout this paper, $0=\lambda_{0}(g)<\lambda_{1}(g) \leq$ $\lambda_{2}(g) \leq \cdots \rightarrow \infty$ denotes the eigenvalues of the minus Laplacian $-\Delta=-\operatorname{tr}$ Hess acting on functions counted with multiplicities. For $p=0,1, \ldots, n$, let

$$
\lambda_{1}\left(\Delta_{C, p}\right):=\inf \left\{\frac{\|\nabla \omega\|_{L^{2}}^{2}}{\|\omega\|_{L^{2}}^{2}}: \omega \in \Gamma\left(\bigwedge^{p} T^{*} M\right) \text { with } \omega \neq 0\right\} .
$$

For metric space $(X, d)$ and $k \in \mathbb{R}_{\geq 0}$, let $\mathcal{H}^{k}$ denote the $k$-dimensional Hausdorff measure. If $0<\mathcal{H}^{k}(X)<\infty$, let $\underline{\mathcal{H}}^{k}$ denote the normalized $k$-dimensional Hausdorff measure:

$$
\underline{\mathcal{H}}^{k}:=\frac{1}{\mathcal{H}^{k}(X)} \mathcal{H}^{k}
$$

In this article, for metric spaces $\left(X_{i}, d_{i}\right)(i=1,2)$, let $d_{1} \times d_{2}$ denote the distance on $X_{1} \times X_{2}$ satisfying

$$
\left(d_{1} \times d_{2}\right)^{2}\left(\left(x_{1}, x_{2}\right),\left(y_{1}, y_{2}\right)\right)=d_{1}^{2}\left(x_{1}, y_{1}\right)+d_{2}^{2}\left(x_{2}, y_{2}\right)
$$

for $\left(x_{1}, x_{2}\right),\left(y_{1}, y_{2}\right) \in X_{1} \times X_{2}$. 


\section{$2.2 \quad$ Metric measure spaces}

In this article we only consider a compact metric measure space with full support and unit total mass for simplicity of the description because it is enough for our purpose.

Definition 2.1. In this article we say that $(X, d, \mathfrak{m})$ is a compact metric measure space if $(X, d)$ is a compact metric space and $\mathfrak{m}$ is a Borel measure with supp $\mathfrak{m}=X$ and $\mathfrak{m}(X)=1$.

We introduce some functional analytic tools on a metric measure space. Our main references are $[2,15,16]$.

Definition 2.2. Let $(X, d, \mathfrak{m})$ be a compact metric measure space.

- Local Lipschitz constant. Let $\operatorname{LIP}(X)$ denote the set of the Lipschitz functions on $X$. For each $f \in \operatorname{LIP}(X)$ and $x \in X$, we define a local Lipschitz constant $\operatorname{Lip}(f)(x)$ by

$$
\operatorname{Lip}(f)(x):=\limsup _{y \rightarrow x} \frac{|f(x)-f(y)|}{d(x, y)}
$$

if $x \in X$ is not an isolated point, and $\operatorname{Lip}(f)(x)=0$ otherwise.

- Cheeger energy. For each $f \in L^{2}(X)$, we define the Cheeger energy $\operatorname{Ch}(f) \in[0, \infty]$ by

$$
\operatorname{Ch}(f):=\frac{1}{2} \inf \left\{\liminf _{i \rightarrow \infty} \int_{X}\left(\operatorname{Lip}\left(f_{i}\right)\right)^{2} \mathrm{dm}: f_{i} \in \operatorname{LIP}(X) \text { and } \lim _{i \rightarrow \infty}\left\|f-f_{i}\right\|_{L^{2}}=0\right\} .
$$

Define

$$
W^{1,2}(X)=W^{1,2}(X, d, \mathfrak{m}):=\left\{f \in L^{2}(X): \operatorname{Ch}(f)<\infty\right\} .
$$

We have that $W^{1,2}(X)$ is a Banach space with the norm $\|f\|_{W^{1,2}}=\left(\|f\|_{L^{2}}^{2}+2 \mathrm{Ch}(f)\right)^{1 / 2}$.

- Minimal relaxed gradient. We say that $|D f| \in L^{2}(X)$ is the minimal relaxed gradient of $f \in W^{1,2}(X)$ if there exists a sequence $\left\{f_{i}\right\}_{i=1}^{\infty}$ of Lipschitz function such that $\lim _{i \rightarrow \infty} \| f-$ $f_{i}\left\|_{L^{2}}=0, \lim _{i \rightarrow \infty}\right\||D f|-\operatorname{Lip}\left(f_{i}\right) \|_{L^{2}}=0$ and

$$
\operatorname{Ch}(f)=\frac{1}{2} \int_{X}|D f|^{2} \mathrm{dm}
$$

For any $f \in W^{1,2}(X)$, the minimal relaxed gradient $|D f| \in L^{2}(X)$ exists and unique. See [2, Definition 4.2 and Lemma 4.3].

- Sobolev-to-Lipschitz property. We say that $(X, d, \mathfrak{m})$ satisfies the Sobolev-to-Lipschitz property if any $f \in W^{1,2}(X)$ with $|D f| \leq 1$ m-a.e. in $X$ is a 1-Lipschitz function on $X$ (more precisely, $f$ has a 1-Lipschitz representative).

- Infinitesimally Hilbertian. We say that $(X, d, \mathfrak{m})$ is infinitesimally Hilbertian if $\mathrm{Ch}$ is a quadratic form. This condition holds if and only if $\left(W^{1,2}(X),\|\cdot\|_{W^{1,2}}\right)$ is a Hilbert space. In this case, we define $\mathcal{E}: W^{1,2}(X) \times W^{1,2}(X) \rightarrow \mathbb{R}$ by

$$
\mathcal{E}(f, g)=\frac{1}{2}(\mathrm{Ch}(f+g)-\mathrm{Ch}(f-g))
$$

- Laplacian. If $(X, d, \mathfrak{m})$ is infinitesimally Hilbertian, then we define

$$
\mathcal{D}(\Delta):=\left\{f \in W^{1,2}(X): \begin{array}{l}
\text { there exists } \Delta f \in L^{2}(X) \text { such that we have } \\
\mathcal{E}(f, g)=-\int_{X} g \Delta f \mathrm{~d} \mathfrak{m} \text { for any } g \in W^{1,2}(X)
\end{array}\right\} .
$$

For each $f \in \mathcal{D}(\Delta), \Delta f \in L^{2}(X)$ is uniquely determined. 
- The function $\left\langle D f_{1}, D f_{2}\right\rangle$. If $(X, d, \mathfrak{m})$ is infinitesimally Hilbertian, then we define $\left\langle D f_{1}, D f_{2}\right\rangle \in L^{1}(X)$ for $f_{1}, f_{2} \in W^{1,2}(X)$ by

$$
\left\langle D f_{1}, D f_{2}\right\rangle=\lim _{\epsilon \rightarrow 0} \frac{1}{2 \epsilon}\left(\left|D\left(f_{1}+\epsilon f_{2}\right)\right|^{2}-\left|D f_{1}\right|^{2}\right) \in L^{1}(X) .
$$

This notion is well-defined by the convexity of the minimal relaxed gradient (see [3, Definition 4.12]). We have that $\left\langle D f_{1}, D f_{1}\right\rangle=\left|D f_{1}\right|^{2} \mathfrak{m}$-a.e. in $X$, that $\left|\left\langle D f_{1}, D f_{2}\right\rangle\right| \leq\left|D f_{1}\right|\left|D f_{2}\right|$ $\mathfrak{m}$-a.e. in $X$, that $\langle\cdot, \cdot\rangle$ is a symmetric bilinear form, and that

$$
\mathcal{E}\left(f_{1}, f_{2}\right)=\int_{X}\left\langle D f_{1}, D f_{2}\right\rangle \mathrm{d} \mathfrak{m}
$$

by [3, Propositions 4.13 and 4.14, Theorem 4.18].

- Heat flow. Let $(X, d, \mathfrak{m})$ be infinitesimally Hilbertian. Let $\left\{P_{t} f\right\}_{t>0}$ denote the gradient flow of the Cheeger energy Ch starting from $f \in L^{2}(X)$ (see [16, Definition 5.2.5]). The flow $\left\{P_{t} f\right\}_{t>0}$ is called the heat flow and characterized as the unique $C^{1}$ map $(0, \infty) \rightarrow L^{2}(X)$ (it turns out to be $C^{\infty}$ [16, Proposition 5.2.12]) satisfying the following conditions (see [16, Theorem 5.1.12]):

- We have $P_{t} f \rightarrow f$ strongly in $L^{2}(X)$ as $t \rightarrow 0$.

- For each $t>0$, we have that $P_{t} f \in \mathcal{D}(\Delta)$ and that

$$
\frac{\mathrm{d}}{\mathrm{d} t} P_{t} f=\Delta P_{t} f
$$

in $L^{2}(X)$.

Moreover, we have the following properties (see [16, Section 5.2.2]):

- For each $t>0$ and $f \in L^{2}(X)$, we have

$$
\begin{aligned}
& \operatorname{Ch}\left(P_{t} f\right) \leq \inf _{g \in W^{1,2}(X)}\left(\operatorname{Ch}(g)+\frac{\|f-g\|_{L^{2}}^{2}}{2 t}\right), \\
& \left\|\Delta P_{t} f\right\|_{L^{2}}^{2} \leq \inf _{g \in \mathcal{D}(\Delta)}\left(\|\Delta g\|_{L^{2}}^{2}+\frac{\|f-g\|_{L^{2}}^{2}}{t^{2}}\right) .
\end{aligned}
$$

See also [16, Theorem 5.1.12].

- For each $t>0, P_{t}: L^{2}(X) \rightarrow L^{2}(X)$ is a linear map satisfying

$$
\int_{X} g P_{t} f \mathrm{~d} \mathfrak{m}=\int_{X} f P_{t} g \mathrm{~d} \mathfrak{m}
$$

for any $f, g \in L^{2}(X)$.

- For each $s, t>0$, we have $P_{s+t}=P_{s} \circ P_{t}$.

- For each $f \in \mathcal{D}(\Delta)$ and $s>0$, we have that

$$
\lim _{t \rightarrow 0+} \frac{P_{t} f-f}{t}=\Delta f
$$

in $L^{2}(X)$ and that $\Delta P_{s} f=P_{s} \Delta f$.

- For each $t>0, c \in \mathbb{R}$ and $f \in L^{2}(X)$ with $f \leq c \mathfrak{m}$-a.e. in $X$, we have $P_{t} f \leq c \mathfrak{m}$-a.e. in $X$. 
- For each $t>0, p \in[1, \infty)$ and $f \in L^{2}(X) \cap L^{p}(X)$, we have $\left\|P_{t} f\right\|_{L^{p}} \leq\|f\|_{L^{p}}$. In particular, we can extend the map $P_{t}: L^{2}(X) \cap L^{p}(X) \rightarrow L^{2}(X) \cap L^{p}(X)$ to $P_{t}$ : $L^{p}(X) \rightarrow L^{p}(X)$.

We can also show the following properties by the above properties:

- For each $f \in W^{1,2}(X)$, we have $\operatorname{Ch}\left(P_{t} f-f\right) \rightarrow 0$ as $t \rightarrow 0$. Indeed, the properties $\mathrm{Ch}\left(P_{t} f\right) \leq \mathrm{Ch}(f)$ and $P_{t} f \rightarrow f$ in $L^{2}$ as $t \rightarrow 0$ imply that $P_{t} f$ converges to $f$ weakly in $W^{1,2}$, and so $\limsup _{t \rightarrow 0} \mathrm{Ch}\left(P_{t} f\right) \leq \mathrm{Ch}(f)$ implies that $P_{t} f$ converges to $f$ strongly in $W^{1,2}$ as $t \rightarrow 0$.

- For each $p \in[1, \infty)$ and $f \in L^{p}(X)$, we have $\left\|P_{t} f-f\right\|_{L^{p}} \rightarrow 0$ as $t \rightarrow 0$. This can be verified by applying the above properties to the truncated function $f_{1}=$ $\max \{\min \{f, c\},-c\}$ for a sufficiently large constant $c>0$ and its remaining part $f-f_{1}$.

- Test functions. Let $(X, d, \mathfrak{m})$ be infinitesimally Hilbertian. We define

$$
\operatorname{TestF}(X):=\left\{f \in \mathcal{D}(\Delta) \cap L^{\infty}(X):|\nabla f| \in L^{\infty}(X) \text { and } \Delta f \in W^{1,2}(X)\right\} .
$$

- Pre-cotangent module. We define

$$
\mathrm{PCM}:=\left\{\left\{\left(f_{i}, A_{i}\right)\right\}: \begin{array}{l}
\left\{A_{i}\right\}_{i=1}^{\infty} \text { is a pairwise disjoint family of Borel subsets of } X \\
\text { with } \bigcup_{i} A_{i}=X, f_{i} \in W^{1,2}(X) \text { with } \sum_{i} \int_{A_{i}}\left|D f_{i}\right|^{2} \mathrm{dm}<\infty
\end{array}\right\} .
$$

We say that $\left\{\left(f_{i}, A_{i}\right)\right\} \in \mathrm{PCM}$ is equivalent to $\left\{\left(g_{i}, B_{i}\right)\right\} \in \mathrm{PCM}$ (denote it by $\left\{\left(f_{i}, A_{i}\right)\right\} \sim$ $\left.\left\{\left(g_{i}, B_{i}\right)\right\}\right)$ if

$$
\left|D\left(f_{i}-g_{j}\right)\right|=0 \text { m-a.e. in } A_{i} \cap B_{j} \text { for each } i, j \in \mathbb{Z}_{>0} .
$$

We define

$$
\begin{aligned}
& |\cdot|: \mathrm{PCM} / \sim \rightarrow L^{2}(X), \quad\left[\left\{\left(f_{i}, A_{i}\right)\right\}\right] \mapsto \sum_{i=1}^{\infty} \chi_{A_{i}}\left|D f_{i}\right|, \\
& \|\cdot\|_{L^{2}}: \mathrm{PCM} / \sim \rightarrow[0, \infty), \quad\left[\left\{\left(f_{i}, A_{i}\right)\right\}\right] \mapsto\left\|\left|\left[\left\{\left(f_{i}, A_{i}\right)\right\}\right]\right|\right\|_{L^{2}}=\left(\sum_{i} \int_{A_{i}}\left|D f_{i}\right|^{2} \mathrm{~d} \mathfrak{m}\right)^{1 / 2},
\end{aligned}
$$

where $\chi_{A_{i}}$ denotes the characteristic function. Then, $\left(\mathrm{PCM} / \sim,\|\cdot\|_{L^{2}}\right)$ is naturally equipped with the structure of the normed vector space. Moreover, we define

$$
\left(\sum_{i=1}^{\infty} a_{i} \chi_{A_{i}}\right) \cdot\left[\left\{\left(f_{i}, B_{i}\right)\right\}\right]:=\left[\left\{\left(a_{i} f_{j}, A_{i} \cap B_{j}\right)\right\}_{i, j}\right]
$$

for each $\sum_{i=1}^{\infty} a_{i} \chi_{A_{i}} \in \operatorname{Sf}(X)$ and $\left[\left\{\left(f_{i}, B_{i}\right)\right\}\right] \in \mathrm{PCM} / \sim$, where $\operatorname{Sf}(X)$ is defined by

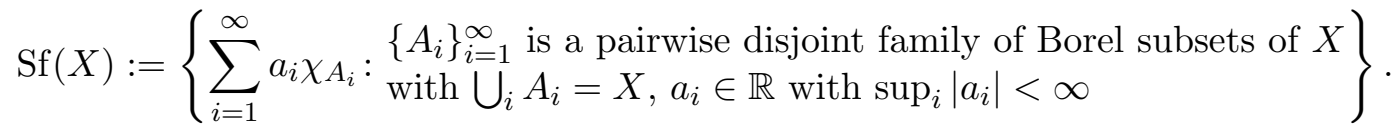

Then, we have $\|f \cdot \omega\|_{L^{2}} \leq\|f\|_{L^{\infty}}\|\omega\|_{L^{2}}$ for each $f \in \operatorname{Sf}(X)$ and $\omega \in \mathrm{PCM} / \sim$.

- Cotangent module. We define the cotangent module $L^{2}\left(T^{*} X\right)$ as a completion of the normed vector space $\left(\mathrm{PCM} / \sim,\|\cdot\|_{L^{2}}\right)$. We can extend the action $\operatorname{Sf}(X) \times \operatorname{PCM} / \sim \rightarrow$ $\mathrm{PCM} / \sim$ to $L^{\infty}(X) \times L^{2}\left(T^{*} X\right) \rightarrow L^{2}\left(T^{*} X\right)$, and $|\cdot|: \mathrm{PCM} / \sim \rightarrow L^{2}(X)$ to $|\cdot|: L^{2}\left(T^{*} X\right) \rightarrow$ 
$L^{2}(X)$. Then, $L^{2}\left(T^{*} X\right)$ is equipped with the structure of an $L^{2}$-normed $L^{\infty}(X)$ module, i.e., we have that

$$
\begin{aligned}
& (f g) \cdot \omega=f \cdot(g \cdot \omega), \quad 1 \cdot \omega=\omega, \quad|\omega| \geq 0 \quad \text { m-a.e. in } \mathrm{X}, \\
& \|\omega\|_{L^{2}}=\||\omega|\|_{L^{2}}, \quad|f \cdot \omega|=|f||\omega|
\end{aligned}
$$

for each $f, g \in L^{\infty}(X)$ and $\omega \in L^{2}\left(T^{*} X\right)$. Note that we use $W^{1,2}(X)$ instead of the Sobolev class $S^{2}(X)$ (see [15, Definition 2.1.4]) in the definition of PCM. However, we can approximate elements of $S^{2}(X)$ by elements of $W^{1,2}(X)$ (see [15, Proposition 2.2.5]), and so our definition of $L^{2}\left(T^{*} X\right)$ coincides with [15, Definition 2.2.1]. In general, $L^{2}\left(T^{*} X\right)$ does not need to be a Hilbert space, and $L^{2}\left(T^{*} X\right)$ is a Hilbert space if and only if $(X, d, \mathfrak{m})$ is infinitesimally Hilbertian [15, Proposition 2.3.17].

- Pointwise scalar product. If $(X, d, \mathfrak{m})$ is infinitesimally Hilbertian, we can define a pointwise scalar product

$$
\left\langle\left[\left\{\left(f_{i}, A_{i}\right)\right\}\right],\left[\left\{\left(g_{i}, B_{i}\right)\right\}\right]\right\rangle:=\sum_{i, j=1}^{\infty} \chi_{A_{i} \cap B_{j}}\left\langle D f_{i}, D g_{j}\right\rangle
$$

for each $\left[\left\{\left(f_{i}, A_{i}\right)\right\}\right],\left[\left\{\left(g_{i}, B_{i}\right)\right\}\right] \in \mathrm{PCM} / \sim$ and extend it to $\langle\cdot, \cdot\rangle: L^{2}\left(T^{*} X\right) \times L^{2}\left(T^{*} X\right) \rightarrow$ $L^{1}(X)$. Then, $\langle\cdot, \cdot\rangle$ is symmetric and $L^{2}\left(T^{*} X\right)$ is a Hilbert space with the inner product defined by

$$
(\omega, \eta) \mapsto \int_{X}\langle\omega, \eta\rangle \mathrm{dm}
$$

for each $\omega, \eta \in L^{2}\left(T^{*} X\right)$. Clearly, for each $\omega, \eta \in L^{2}\left(T^{*} X\right)$, we have $\langle\omega, \omega\rangle=|\omega|^{2}$ and $|\langle\omega, \eta\rangle| \leq|\omega||\eta| \mathfrak{m}$-a.e. in $X$.

- Differential. We define the differential d: $W^{1,2}(X) \rightarrow L^{2}\left(T^{*} X\right)$ by $\mathrm{d} f=[(f, X)]$ for each $f \in W^{1,2}(X)$. Clearly, we have that $|D f|=|\mathrm{d} f| \in L^{2}(X)$ for each $f \in W^{1,2}(X)$. Moreover, if $(X, d, \mathfrak{m})$ is infinitesimally Hilbertian, then we have $\langle D f, D g\rangle=\langle\mathrm{d} f, \mathrm{~d} g\rangle \in L^{1}(X)$ for each $f, g \in W^{1,2}(X)$.

- Tangent module. We define the tangent module $L^{2}(T X)$ by

$$
L^{2}(T X):=\left\{\begin{array}{l}
V: L^{2}\left(T^{*} X\right) \rightarrow L^{1}(X) \text { is a bounded linear operator such that } \\
V(f \cdot \omega)=f V(\omega) \text { holds for all } f \in L^{\infty}(X) \text { and } \omega \in L^{2}\left(T^{*} X\right)
\end{array}\right\} .
$$

If $(X, d, \mathfrak{m})$ is infinitesimally Hilbertian, then the map

$$
L^{2}\left(T^{*} X\right) \rightarrow L^{2}(T X), \quad \omega \mapsto\langle\omega, \cdot\rangle
$$

is bijective (see [15, Theorem 1.2.24]). Under this identification, $L^{2}(T X)$ is equipped with the structure of an $L^{2}$-normed $L^{\infty}(M)$ module and a pointwise scalar product $\langle\cdot, \cdot\rangle: L^{2}(T X) \times L^{2}(T X) \rightarrow L^{1}(X)$. Note that even if $(X, d, \mathfrak{m})$ is not infinitesimally Hilbertian, the tangent module $L^{2}(T X)$ is naturally equipped with the structure of an $L^{2}$ normed $L^{\infty}(M)$ module (see [15, Definition 1.2.6 and Proposition 1.2.14]).

- Gradient. If $(X, d, \mathfrak{m})$ is infinitesimally Hilbertian, we define a map $\nabla: W^{1,2}(X) \rightarrow L^{2}(T X)$ by $\nabla f=\langle\mathrm{d} f, \cdot\rangle$ for each $f \in W^{1,2}(X)$. Clearly, we have that $|\nabla f|=|D f|=|\mathrm{d} f| \in L^{2}(X)$ and $\langle\nabla f, \nabla g\rangle=\langle D f, D g\rangle \in L^{1}(X)$ for each $f, g \in W^{1,2}(X)$.

- Divergence. If $(X, d, \mathfrak{m})$ is infinitesimally Hilbertian, then we define

$$
\mathcal{D}(\text { div }):=\left\{V \in L^{2}(T X): \begin{array}{l}
\text { there exists } \operatorname{div} V \in L^{2}(X) \text { such that we have } \\
\int_{X}\langle V, \nabla g\rangle \mathrm{d} \mathfrak{m}=-\int_{X} g \operatorname{div} V \mathrm{dm} \text { for any } g \in W^{1,2}(X)
\end{array}\right\} .
$$

For each $V \in \mathcal{D}(\operatorname{div})$, div $V \in L^{2}(X)$ is uniquely determined. 
- Symmetric part of the covariant derivative [7, Definition 5.4]. Let $(X, d, \mathfrak{m})$ be infinitesimally Hilbertian. For a vector field $V \in \mathcal{D}\left(\right.$ div) we write $D^{\text {sym }} V \in L^{2}(X)$ if there exists $c>0$ such that we have

$$
\left|\int_{X}\langle V, \nabla f\rangle \Delta g+\langle V, \nabla g\rangle \Delta f-\langle\nabla f, \nabla g\rangle \operatorname{div} V \operatorname{dm}\right| \leq c\|\nabla f\|_{L^{4}}\|\nabla g\|_{L^{4}}
$$

for any $f, g \in \mathcal{D}(\Delta)$ with $|\nabla f|,|\nabla g| \in L^{4}(X)$ and $\Delta f, \Delta g \in L^{4}(X)$.

\subsection{The RCD* condition and some properties}

In this subsection we recall the definition of the $\operatorname{RCD}^{*}(K, N)$ space and its properties.

Definition 2.3. We say that an infinitesimally Hilbertian metric measure space $(X, d, \mathfrak{m})$ satisfies the Bakry-Émery condition $\operatorname{BE}(K, N)$ with $K \in \mathbb{R}$ and $N \in[1, \infty)$ if for all $u \in \mathcal{D}(\Delta)$ with $\Delta u \in W^{1,2}(X)$ and all $\phi \in \mathcal{D}(\Delta) \cap L^{\infty}(X)$ with $\phi \geq 0$ and $\Delta \phi \in L^{\infty}(X)$, we have

$$
\frac{1}{2} \int_{X} \Delta \phi|\nabla u|^{2} \mathrm{~d} \mathfrak{m} \geq \int_{X} \phi\left(\langle\nabla \Delta u, \nabla u\rangle+K|\nabla u|^{2}+\frac{1}{N}(\Delta u)^{2}\right) \mathrm{d} \mathfrak{m} .
$$

Definition 2.4. We say that an infinitesimally Hilbertian metric measure space $(X, d, \mathfrak{m})$ satisfies the Bakry-Ledoux condition $\operatorname{BL}(K, N)$ with $K \in \mathbb{R}$ and $N \in[1, \infty)$ if for all $u \in W^{1,2}(X)$ and $t>0$ we have

$$
\left|\nabla P_{t} f\right|^{2}+\frac{2 t C(t)}{N}\left|\Delta P_{t} f\right|^{2} \leq \mathrm{e}^{-2 K t} P_{t}\left(|\nabla f|^{2}\right)
$$

m-a.e. in $\mathrm{X}$, where $C(t)>0$ is a function satisfying $C(t)=1+O(t)$ as $t \rightarrow 0$.

Theorem 2.5 ([14, Theorem 4.8 and Proposition 4.9]). An infinitesimally Hilbertian metric measure space $(X, d, m)$ satisfies the $\mathrm{BE}(K, N)$ condition if and only if it satisfies the $\mathrm{BL}(K, N)$ condition.

Let us recall an equivalent version of the definition of the $\operatorname{RCD}^{*}(K, N)$ condition (see [14, Theorem 7 and Definition 3.16] for the equivalence). If the total mass is finite, the $\operatorname{RCD}^{*}(K, N)$ condition is equivalent to the $\operatorname{RCD}(K, N)$ condition by [10, Corollary 13.7].

Definition 2.6. We say that a compact infinitesimally Hilbertian metric measure space $(X, d, \mathfrak{m})$ satisfies the $\operatorname{RCD}^{*}(K, N)$ condition with $K \in \mathbb{R}$ and $N \in[1, \infty)$ if $(X, d, \mathfrak{m})$ satisfies the $\mathrm{BE}(K, N)$ condition and the Sobolev-to-Lipschitz property.

For more general metric measure space, we add the volume growth assumption to Definition 2.6. However, it is automatically satisfied in our situation because we assume that $\mathfrak{m}(X)=1$. Note that Definition 2.6 implies that $(X, d)$ is a geodesic space by [4, Theorems 3.9 and 3.10] and [9, Theorem 2.5.23]. The original definition also implies this property (see [14, Remark 3.8] and [31, Remark 4.6]).

The definition of the $\operatorname{RCD}^{*}(K, N)$ condition is consistent to the smooth case.

Proposition 2.7 ([14, Proposition 4.21]). For any n-dimensional closed Riemannian manifold $(M, g)$ and real numbers $K \in \mathbb{R}$ and $N \in[1, \infty)$, we have that $\left(M, d_{g}, \underline{\mathcal{H}}^{n}\right)$ satisfies the $\operatorname{RCD}^{*}(K, N)$ condition if and only if Ric $\geq K g$ and $n \leq N$ hold.

Let us compare the local Lipschitz constant and the minimal relaxed gradient for Lipschitz functions. If the $\operatorname{RCD}^{*}(K, N)$ condition holds, then we have the doubling condition [32, Corollary 2.4] and the weak Poincaré inequality [28, Theorem 1.1]. Moreover, our minimal relaxed gradient coincides with Cheeger's minimal generalized upper gradient [11, Definition 2.9] by [2, Theorem 6.2]. Thus, we have the following theorem by [11, Theorem 6.1]: 
Theorem 2.8. Let $(X, d, \mathfrak{m})$ be a compact metric measure space satisfying the $\operatorname{RCD}^{*}(K, N)$ condition. Then, for any $f \in \operatorname{LIP}(X)$, we have $\operatorname{Lip} f=|\nabla f| \mathfrak{m}$-a.e. in $X$.

Under the $\operatorname{RCD}^{*}(K, N)$ condition, we have that

$$
\operatorname{TestF}(X)=\left\{f \in \mathcal{D}(\Delta) \cap \operatorname{LIP}(X): \Delta f \in W^{1,2}(X)\right\} .
$$

Let us make a remark on the heat kernel. Let $(X, d, \mathfrak{m})$ be a compact metric measure space satisfying the $\operatorname{RCD}^{*}(K, N)$ condition. Then, $\mathcal{E}$ is a strongly local Dirichlet form on $(X, \mathfrak{m})$ by [2, Proposition 4.8] and [3, Proposition 4.11], and we have

$$
d(x, y)=\sup \{|f(x)-f(y)|: f \in \operatorname{LIP}(X) \text { with }|\nabla f| \leq 1 \mathfrak{m} \text {-a.e. in } X\}
$$

by [4, Theorem 3.9]. Since we have the doubling condition by [32, Corollary 2.4] and the strong local $(2,2)$ Poincaré inequality [30, Property (Ic)] by [28, Theorem 1.1] and [19, Theorem 1], we can apply [29, Proposition 2.3] and [30, Proposition 3.1] (see also [30, Theorem 3.5]), and so there exists a locally Hölder continuous function $p:(0, \infty) \times X \times X \rightarrow \mathbb{R}$ such that

$$
P_{t} f(x)=\int_{X} p(t, x, y) f(y) \mathrm{d} \mathfrak{m}(y)
$$

holds for any $f \in L^{1}(X)$. By [23, Theorem 1.2], for any $\epsilon>0$, there exist constants $C_{i}=$ $C_{i}(\epsilon, K, N)>1$ such that

$$
\frac{C_{1}^{-1}}{\mathfrak{m}\left(B_{\sqrt{t}}(x)\right)} \exp \left(-\frac{d^{2}(x, y)}{(4-\epsilon) t}-C_{2} t\right) \leq p(t, x, y) \leq \frac{C_{1}}{\mathfrak{m}\left(B_{\sqrt{t}}(x)\right)} \exp \left(-\frac{d^{2}(x, y)}{(4+\epsilon) t}+C_{2} t\right)
$$

holds for each $x, y \in X$ and $t>0$. Here, we defined $B_{r}(x):=\{z \in X: d(x, z)<r\}$ for $x \in X$ and $r>0$. By this and the Bishop-Gromov inequality [32, Theorem 2.3], we have the following:

- For any $f \in L^{1}(X)$, we have

$$
\left\|P_{t} f\right\|_{L^{\infty}} \leq C(t)\|f\|_{L^{1}} .
$$

- For any $f \in C(X)$, the function

$$
[0, \infty) \times X \rightarrow \mathbb{R}, \quad(t, x) \mapsto\left(P_{t} f\right)(x)
$$

is continuous. Here, we defined $P_{0} f:=f$.

For any $f \in W^{1,2}(X)$ and $t>0$, we have $P_{t} f \in \operatorname{LIP}(X)$ by $(2.2)$ for $|\nabla f|^{2} \in L^{1}(X)$, the $\mathrm{BL}(K, N)$ condition and the Sobolev-to-Lipschitz property, and so $P_{t} f \in \operatorname{TestF}(X)$ by $\Delta P_{t} f=$ $P_{t / 2} \Delta P_{t / 2} f \in W^{1,2}(X)$. In particular, $\operatorname{TestF}(X) \subset W^{1,2}(X)$ is dense. As a corollary, we have $P_{t} f=P_{t / 2} P_{t / 2} f \in \operatorname{TestF}(X)$ for any $f \in L^{2}(X)$ and $t>0$. Note that since we assumed that $(X, d, \mathfrak{m})$ is compact and $\mathfrak{m}(X)=1$, we can skip the truncation procedure.

We next recall some basic facts about the spectrum of $-\Delta$ on a compact metric measure space $(X, d, \mathfrak{m})$ satisfying the $\operatorname{RCD}^{*}(K, N)$ condition. By [28, Theorem 1.1], [19, Theorem 1] and [20, Theorem 8.1], the inclusion $W^{1,2}(X) \rightarrow L^{2}(X)$ is a compact operator. Thus, the spectrum of $-\Delta$ is discrete and positive:

$$
0=\lambda_{0}<\lambda_{1} \leq \lambda_{2} \leq \cdots \rightarrow \infty
$$

as the smooth case. See also the proof of [14, Theorem 4.22]. Let $\left\{\phi_{i}\right\}_{i=0}^{\infty}$ be the corresponding eigenfunctions. Then,

$$
\bigoplus_{i=0}^{\infty} \mathbb{R} \phi_{i}=\left\{\sum_{i=0}^{k} a_{i} \phi_{i}: k \in \mathbb{Z}_{\geq 0} \text { and } a_{i} \in \mathbb{R}(i=0, \ldots, k)\right\}
$$

is dense in $L^{2}(X)$. 
Finally, let us recall the notion of the regular Lagrangian flow, which is a flow for a vector field in a non-smooth setting. Although time dependent vector fields are considered in [6], we only deal with time independent vector fields because it is enough for our purpose.

Definition $2.9([6])$. Let $(X, d, \mathfrak{m})$ be a compact measure space satisfying the $\operatorname{RCD}^{*}(K, N)$ condition and take $T>0$. We say that $\mathrm{Fl}^{V}:[0, T] \times X \rightarrow X$ is a regular Lagrangian flow for a vector field $V \in L^{2}(T X)$ if the following properties hold:

(i) There exists a constant $C>0$ such that

$$
\left(\mathrm{Fl}_{s}^{V}\right)_{*} \mathfrak{m} \leq C \mathfrak{m}
$$

holds for any $s \in[0, T]$.

(ii) For each $x \in X$, the curve

$$
[0, T] \rightarrow X, \quad s \mapsto \mathrm{Fl}_{s}^{V}(x)
$$

is continuous and $\mathrm{Fl}_{0}^{V}=\operatorname{Id}_{X}$. Moreover, the map $X \rightarrow C([0, T] ; X), x \mapsto \mathrm{Fl}^{V}(\cdot, x)$ is Borel measurable. Here, $C([0, T] ; X)$ is equipped with the topology induced by the uniformly convergence.

(iii) For any Lipschitz function $f$ on $X$, we have that $f\left(\mathrm{Fl}^{V}(\cdot, x)\right) \in W^{1,1}(0, T)$ for $\mathfrak{m}$-a.e. $x \in X$ and

$$
\frac{\mathrm{d}}{\mathrm{d} t} f\left(\left(\mathrm{Fl}^{V}(t, x)\right)=\mathrm{d} f(V)\left(\left(\mathrm{Fl}^{V}(t, x)\right)\right.\right.
$$

for $\mathcal{L}^{1} \times \mathfrak{m}$-a.e. $(t, x) \in(0, T) \times X$.

Note that the Borel measurability in (ii) can be verified under the assumption of the following existence theorem because we construct the flow using the disintegration theorem in $[6$, Theorem 8.4] and [7, Theorem 7.8].

We use the following form of the result of [6]. See also [7].

Theorem 2.10 (Ambrosio-Trevisan [6]). Let $(X, d, \mathfrak{m})$ be a metric measure space satisfying the $\operatorname{RCD}^{*}(K, N)$ condition and take $T>0$. For any vector field $V \in \mathcal{D}(\operatorname{div})$ with $D^{\mathrm{sym}} V \in L^{2}(X)$ and $(\operatorname{div} V)^{-} \in L^{\infty}(X)\left((\operatorname{div} V)^{-}\right.$denotes the negative part of the divergence $\left.\operatorname{div} V\right)$, a regular

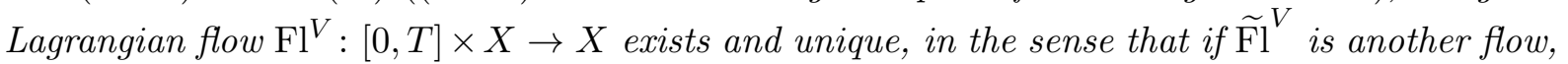
then for $\mathfrak{m}$-a.e. $x \in X$ we have that $\mathrm{Fl}^{V}(s, x)=\widetilde{\mathrm{Fl}}^{V}(s, x)$ for every $s \in[0, T]$. Moreover, we have that

$$
\left(\mathrm{Fl}_{s}^{V}\right)_{*} \mathfrak{m} \leq \exp \left(T\left\|(\operatorname{div} V)^{-}\right\|_{L^{\infty}}\right) \mathfrak{m}
$$

for all $s \in[0, T]$.

Remark 2.11. Let us give some comments about which assertions in [6] correspond to Theorem 2.10. We set $\mathcal{A}:=\operatorname{LIP}(X)$. The concept of the regular Lagrangian flow is closely related to the continuity equation

$$
\frac{\mathrm{d}}{\mathrm{d} t} u_{t}+\operatorname{div}\left(u_{t} V\right)=0 .
$$

For $\bar{u} \in L^{\infty}(X)$ with $\bar{u} \geq 0$, there exists a weakly continuous weak solution (in the duality with $\mathcal{A}) u \in L^{\infty}\left([0, T] ; L^{\infty}(X)\right)$ of the continuity equation with initial condition $\bar{u}$ in the sense of $[6$, Definition 4.2$]$ satisfying

$$
\left\|u_{t}\right\|_{L^{\infty}} \leq\|\bar{u}\|_{L^{\infty}} \exp \left(T\left\|(\operatorname{div} V)^{-}\right\|_{L^{\infty}}\right)
$$


and $u_{t} \geq 0$ for each $t \in[0, T]$ by [6, Theorems 4.3 and 4.6] (see also [7, Theorem 6.1]). Note that [6, Theorem 4.6] deals with approximated solutions. However, since we get the solution of the continuity equation as a weak* limit of them, we have the same estimate. Moreover, since we have the $L^{4}-\Gamma$ inequality [6, Definition 5.1 and Corollary 6.3], the solution is unique by [6, Theorem 5.4] (see also [7, Theorem 6.4]) putting $p=s=r=4$ and $q=2$. Thus, by [6, Theorem 9.2], we can apply [7, Theorem 7.7] (see also [6, Theorem 8.3]), and so there exists a unique regular Lagrangian flow for $V$. Moreover, its proof shows the estimate (2.3).

\subsection{Gromov-Hausdorff convergence and functions}

In this subsection we recall some properties about the Gromov-Hausdorff convergence.

Definition 2.12 (Hausdorff distance). Let $(X, d)$ be a metric space. For each point $x_{0} \in X$, subsets $A, B \subset X$ and $r>0$, define

$$
\begin{aligned}
& d\left(x_{0}, A\right):=\inf \left\{d\left(x_{0}, a\right): a \in A\right\}, \\
& B_{r}(A):=\{x \in X: d(x, A)<r\}, \\
& d_{\mathrm{H}, d}(A, B):=\inf \left\{\epsilon>0: A \subset B_{\epsilon}(B) \text { and } B \subset B_{\epsilon}(A)\right\} .
\end{aligned}
$$

We call $d_{\mathrm{H}, d}$ the Hausdorff distance.

The Hausdorff distance defines a metric on the collection of compact subsets of $X$.

Definition 2.13 (Gromov-Hausdorff distance). Let $\left(X, d_{X}\right),\left(Y, d_{Y}\right)$ be metric spaces. Define

$$
d_{\mathrm{GH}}(X, Y):=\inf \left\{d_{\mathrm{H}, d}(X, Y): \begin{array}{l}
d \text { is a metric on } X \amalg Y \text { such that } \\
\left.d\right|_{X}=d_{X} \text { and }\left.d\right|_{Y}=d_{Y}
\end{array}\right\} .
$$

The Gromov-Hausdorff distance defines a metric on the set of isometry classes of compact metric spaces (see [27, Proposition 11.1.3]).

Definition 2.14 ( $\epsilon$-Hausdorff approximation map). Let $\left(X, d_{X}\right),\left(Y, d_{Y}\right)$ be metric spaces. We say that a map $\psi: X \rightarrow Y$ is an $\epsilon$-Hausdorff approximation map for $\epsilon>0$ if the following two conditions hold.

(i) For all $a, b \in X$, we have $\left|d_{X}(a, b)-d_{Y}(\psi(a), \psi(b))\right|<\epsilon$,

(ii) $\psi(X)$ is $\epsilon$-dense in $Y$, i.e., for all $y \in Y$, there exists $x \in X$ with $d_{Y}(\psi(x), y)<\epsilon$.

If there exists an $\epsilon$-Hausdorff approximation map from $X$ to $Y$, then we can show that $d_{\mathrm{GH}}(X, Y) \leq 3 \epsilon / 2$. Conversely, if $d_{\mathrm{GH}}(X, Y)<\epsilon$, then there exists a $2 \epsilon$-Hausdorff approximation map from $X$ to $Y$.

Definition 2.15. Suppose that a sequence of $n$-dimensional closed Riemannian manifolds $\left\{\left(M_{i}, g_{i}\right)\right\}_{i \in \mathbb{Z}_{>0}}$ with $\operatorname{Ric}_{i} \geq K g_{i}$ and $\operatorname{diam}\left(M_{i}\right) \leq D(K \in \mathbb{R}, D>0)$ converges to a metric space $(X, d)$ in the Gromov-Hausdorff topology. Fix a sequence of $\epsilon_{i}$-Hausdorff approximation maps $\psi_{i}: M_{i} \rightarrow X$, where $\left\{\epsilon_{i}\right\}$ is some sequence of positive real numbers with $\lim _{i \rightarrow \infty} \epsilon_{i}=0$.

- We say a sequence $\left\{x_{i}\right\}$ with $x_{i} \in M_{i}$ converges to $x \in X$ if $\lim _{i \rightarrow \infty} \psi_{i}\left(x_{i}\right)=x$ in $X$ (denote it by $\left.x_{i} \stackrel{\mathrm{GH}}{\rightarrow} x\right)$.

- Let $\mathfrak{m}$ be a Borel measure on $X$. We say that a sequence $\left\{\left(M_{i}, g_{i}, \underline{\mathcal{H}}^{n}\right)\right\}$ converges to a metric measure space $(X, d, \mathfrak{m})$ in the measured Gromov-Hausdorff topology if

$$
\lim _{i \rightarrow \infty} \underline{\mathcal{H}}^{n}\left(B_{r}\left(x_{i}\right)\right)=\mathfrak{m}\left(B_{r}(x)\right)
$$


holds for any $r>0, x_{i} \in M_{i}$ and $x \in X$ with $x_{i} \stackrel{\text { GH }}{\rightarrow} x$. Note that taking a subsequence, such a limit measure exists by [12, Theorems 1.6 and 1.10]. Moreover, $(X, d, \mathfrak{m})$ satisfies the $\operatorname{RCD}^{*}(K, N)$ condition by [14, Theorem 3.22].

Suppose that a sequence $\left\{\left(M_{i}, g_{i}, \underline{\mathcal{H}}^{n}\right)\right\}$ converges to $(X, d, \mathfrak{m})$ in the measured Gromov-Hausdorff topology.

- We say that $f_{i} \in L^{2}\left(M_{i}\right)\left(i \in \mathbb{Z}_{>0}\right)$ converges to $f \in L^{2}(X)$ strongly at $x \in X[22$, Definition 3.7] if we have that

$$
\begin{aligned}
& \lim _{r \rightarrow 0} \limsup _{i \rightarrow \infty}\left(\frac{1}{\underline{\mathcal{H}}^{n}\left(B_{r}\left(x_{i}\right)\right)} \int_{B_{r}\left(x_{i}\right)}\left|f_{i}-\frac{1}{\mathfrak{m}\left(B_{r}(x)\right)} \int_{B_{r}(x)} f \mathrm{~d} \mathfrak{m}\right| \mathrm{d} \underline{\mathcal{H}}^{n}\right)=0, \\
& \lim _{r \rightarrow 0} \limsup _{i \rightarrow \infty}\left(\frac{1}{\mathfrak{m}\left(B_{r}(x)\right)} \int_{B_{r}(x)}\left|f-\frac{1}{\underline{\mathcal{H}}^{n}\left(B_{r}\left(x_{i}\right)\right)} \int_{B_{r}\left(x_{i}\right)} f_{i} \mathrm{~d} \underline{\mathcal{H}}^{n}\right| \mathrm{d} \mathfrak{m}\right)=0
\end{aligned}
$$

for all $x_{i} \in M_{i}$ with $x_{i} \stackrel{\text { GH }}{\rightarrow} x$.

- We say that $f_{i} \in L^{2}\left(M_{i}\right)\left(i \in \mathbb{Z}_{>0}\right)$ converges to $f \in L^{2}(X)$ weakly in $L^{2}$ ( $L^{2}$ boundedness and weakly convergence [22, Definition 3.4 and Proposition 3.17]) if

$$
\sup _{i}\left\|f_{i}\right\|_{L^{2}}<\infty
$$

and for all $r>0, x_{i} \in M_{i}$ and $x \in X$ with $x_{i} \stackrel{\text { GH }}{\rightarrow} x$, we have

$$
\lim _{i \rightarrow \infty} \int_{B_{r}\left(x_{i}\right)} f_{i} \mathrm{~d} \underline{\mathcal{H}}^{n}=\int_{B_{r}(x)} f \mathrm{~d} \mathfrak{m} .
$$

Note that we have $\|f\|_{L^{2}} \leq \liminf _{i \rightarrow \infty}\left\|f_{i}\right\|_{L^{2}}$ by [22, Proposition 3.29].

- We say that $f_{i} \in L^{2}\left(M_{i}\right)\left(i \in \mathbb{Z}_{>0}\right)$ converges to $f \in L^{2}(X)$ strongly in $L^{2}$ [22, Definition 3.21 and Proposition 3.31] if $f_{i}$ converges to $f$ weakly in $L^{2}$, and

$$
\limsup _{i \rightarrow \infty}\left\|f_{i}\right\|_{L^{2}} \leq\|f\|_{L^{2}}
$$

holds.

- We say that $V_{i} \in L^{2}\left(T M_{i}\right)\left(i \in \mathbb{Z}_{>0}\right)$ converges to $V \in L^{2}(T X)$ weakly in $L^{2}$ [22, Definition 3.42] if

$$
\sup _{i}\left\|V_{i}\right\|_{L^{2}}<\infty
$$

and for all $r>0, y_{i}, z_{i} \in X_{i}$ and $y, z \in X$ with $y_{i} \stackrel{\mathrm{GH}}{\rightarrow} y, z_{i} \stackrel{\mathrm{GH}}{\rightarrow} z$, we have

$$
\lim _{i \rightarrow \infty} \int_{B_{r}\left(y_{i}\right)}\left\langle V_{i}, \nabla r_{z_{i}}\right\rangle \mathrm{d} \underline{\mathcal{H}}^{n}=\int_{B_{r}(y)}\left\langle V, \nabla r_{z}\right\rangle \mathrm{d} \mathfrak{m},
$$

where $r_{z}(x):=d(z, x)$ for each $x \in X$. Note that we have $\|V\|_{L^{2}} \leq \liminf _{i \rightarrow \infty}\left\|V_{i}\right\|_{L^{2}}$ by $[22$, Proposition 3.64].

- We say that $V_{i} \in L^{2}\left(T M_{i}\right)\left(i \in \mathbb{Z}_{>0}\right)$ converges to $X \in L^{2}(T X)$ strongly in $L^{2}[22$, Definition 3.58 and Proposition 3.66] if $V_{i}$ converges to $V$ weakly in $L^{2}$ and

$$
\limsup _{i \rightarrow \infty}\left\|V_{i}\right\|_{2} \leq\|V\|_{2}
$$

holds. 
Proposition 2.16 ([22, Proposition 3.32]). Suppose that a sequence of $n$-dimensional closed Riemannian manifolds $\left\{\left(M_{i}, g_{i}, \underline{\mathcal{H}}^{n}\right)\right\}_{i \in \mathbb{Z}_{>0}}$ with $\operatorname{Ric}_{i} \geq K g_{i}$ and $\operatorname{diam}\left(M_{i}\right) \leq D(K \in \mathbb{R}, D>0)$ converges to a compact metric measure space $(X, d, \mathfrak{m})$ in the measured Gromov-Hausdorff topology. For any $f_{i} \in L^{\infty}\left(M_{i}\right)\left(i \in \mathbb{Z}_{>0}\right)$ with $\sup \left\|f_{i}\right\|_{L^{\infty}}<\infty$ and $f \in L^{\infty}(X)$, the following conditions are mutually equivalent:

(i) $f_{i} \rightarrow f$ strongly at a.e. $x \in X$.

(ii) $f_{i} \rightarrow f$ strongly in $L^{2}$.

Note that the implication $(i) \Rightarrow(i i)$ is a direct consequence of $[22$, Definitions 3.21 and 3.25, Proposition 3.24].

Theorem 2.17 ([22, Theorem 1.3]). Suppose that a sequence of n-dimensional closed Riemannian manifolds $\left\{\left(M_{i}, g_{i}, \underline{\mathcal{H}}^{n}\right)\right\}_{i \in \mathbb{Z}_{>0}}$ with $\operatorname{Ric}_{i} \geq K g_{i}$ and $\operatorname{diam}\left(M_{i}\right) \leq D(K \in \mathbb{R}, D>0)$ converges to a compact metric measure space $(X, d, \mathfrak{m})$ in the measured Gromov-Hausdorff topology. If $f_{i} \in L^{2}\left(M_{i}\right) \cap C^{2}\left(M_{i}\right)\left(i \in \mathbb{Z}_{>0}\right)$ converges to $f \in L^{2}(X)$ weakly in $L^{2}$, and satisfies

$$
\sup _{i \in \mathbb{Z}_{>0}}\left(\left\|f_{i}\right\|_{W^{1,2}}+\left\|\Delta f_{i}\right\|_{L^{2}}\right)<\infty,
$$

then we have the following:

(i) $f_{i} \rightarrow f$ and $\nabla f_{i} \rightarrow \nabla f$ strongly in $L^{2}$,

(ii) $f \in \mathcal{D}\left(\Delta_{X}\right)$ and $\Delta f_{i} \rightarrow \Delta f$ weakly in $L^{2}$.

\subsection{Convergence to the product space $S^{n-p}(1) \times X$}

We summarize several results proven in [1] for later use.

Proposition 2.18 ([1, Proposition 4.17, Lemma 4.22 and Theorem 4.47]). For given integers $n \geq 5$ and $2 \leq p<n / 2$ and a positive real number $\epsilon>0$, there exists $\delta=\delta(n, p, \epsilon)>0$ such that the following properties hold. Let $(M, g)$ be an $n$-dimensional closed Riemannian manifold with $\operatorname{Ric}_{g} \geq(n-p-1) g$. Assume that $\lambda_{n-p+1}(g) \leq n-p+\delta$ and that either $\lambda_{1}\left(\Delta_{C, p}\right) \leq \delta$ or $\lambda_{1}\left(\Delta_{C, n-p}\right) \leq \delta$. Then, for any $f \in \operatorname{Span}_{\mathbb{R}}\left\{f_{1}, \ldots, f_{n-p+1}\right\}$ with $\|f\|_{2}^{2}=1 /(n-p+1)$, we have the following:

(i) There exists a measurable subset $V_{f} \subset M$ such that $\operatorname{Vol}\left(M \backslash V_{f}\right) \leq \epsilon \operatorname{Vol}(M)$ and $\mid f^{2}+$ $|\nabla f|^{2}-1 \mid \leq \epsilon$ holds in $V_{f}$.

(ii) There exists a non-empty compact subset $A_{f} \subset M$ such that $|f(x)-1| \leq \epsilon$ for any $x \in A_{f}$, $\left|f(x)-\cos d\left(x, A_{f}\right)\right| \leq \epsilon$ for any $x \in M$ and $\sup _{x \in M} d\left(x, A_{f}\right) \leq \pi+\epsilon$ hold.

(iii) Define $\widetilde{\Psi}: M \rightarrow \mathbb{R}^{n-p+1}$ by $\widetilde{\Psi}(x)=\left(f_{1}(x), \ldots, f_{n-p+1}(x)\right)(x \in M)$. Then, we have ||$\widetilde{\Psi}(x)|-1| \leq \epsilon$ for any $x \in M$.

(iv) Choose $a_{f}(x) \in A_{f}$ such that $d\left(x, A_{f}\right)=d\left(x, a_{f}(x)\right)$ for each $x \in M$. Then, we have that the map

$$
\Phi_{f}: \quad M \rightarrow S^{n-p}(1) \times A_{f}, \quad x \mapsto\left(\frac{\widetilde{\Psi}(x)}{|\widetilde{\Psi}(x)|}, a_{f}(x)\right)
$$

is an $\epsilon$-Hausdorff approximation.

Proposition 2.19 ([1, Corollary 4.53]). For given integers $n \geq 5$ and $2 \leq p<n / 2$ and a positive real number $\epsilon>0$, there exists $\delta=\delta(n, p, \epsilon)>0$ such that if $(M, g)$ is an $n$-dimensional closed Riemannian manifold with $\operatorname{Ric}_{g} \geq(n-p-1) g, \lambda_{n-p}(g) \leq n-p+\delta$ and $\lambda_{1}\left(\Delta_{C, n-p}\right) \leq \delta$, then we have $\lambda_{n-p+1}(g) \leq n-p+\epsilon$.

If we assume $\lambda_{1}\left(\Delta_{C, p}\right) \leq \delta$ instead of $\lambda_{1}\left(\Delta_{C, n-p}\right) \leq \delta$ in Proposition 2.19, the assertion fails (see [1, Corollary 3.2 and Proposition 3.3]). 


\section{Structure of the limit}

\subsection{Splitting of the measure}

In this subsection we show that there exists a Borel measure $\mathfrak{m}_{X}$ on $\mathrm{X}$ such that $\mathfrak{m}=\underline{\mathcal{H}}^{n-p} \times \mathfrak{m}_{X}$ holds under Assumption 3.1 below.

Assumption 3.1. Take $n \geq 5$ and $2 \leq p<n / 2$. Let $\left\{\left(M_{i}, g_{i}\right)\right\}_{i \in \mathbb{N}}$ be a sequence of $n$-dimensional closed Riemannian manifolds with $\operatorname{Ric}_{g_{i}} \geq(n-p-1) g_{i}$ that satisfies $\lim _{i \rightarrow \infty} \lambda_{n-p+1}\left(g_{i}\right)=n-p$ and either $\lim _{i \rightarrow \infty} \lambda_{1}\left(\Delta_{C, p}, g_{i}\right)=0$ or $\lim _{i \rightarrow \infty} \lambda_{1}\left(\Delta_{C, n-p}, g_{i}\right)=0$. Let $f_{1, i}, \ldots, f_{n-p+1, i}^{i \rightarrow \infty}$ denote the first $n-p+1$ eigenfunctions on $\left(M_{i}, g_{i}\right)$ with $\left\|f_{k, i}\right\|_{L^{2}}^{2}=1 /(n-p+1)(k=1, \ldots, n-p+1)$. Put

$$
\Psi_{i}: \quad M_{i} \rightarrow S^{n-p}(1), \quad x \mapsto \frac{\left(f_{1, i}(x), \ldots, f_{n-p+1, i}(x)\right)}{\left|\left(f_{1, i}(x), \ldots, f_{n-p+1, i}(x)\right)\right|}
$$

Let $X$ be a compact metric space and $\mathfrak{m}$ be a Borel measure on $S^{n-p}(1) \times X$ with unit volume. Suppose that, for each $i$, there exists a map $b_{i}: M_{i} \rightarrow X$ such that the map

$$
\left(\Psi_{i}, b_{i}\right): M_{i} \rightarrow S^{n-p}(1) \times X
$$

is an $\epsilon_{i}$-approximation, where $\left\{\epsilon_{i}\right\}$ is some sequence of positive real numbers with $\epsilon_{i} \rightarrow 0$ as $i \rightarrow \infty$. Suppose that the sequence $\left\{\left(M_{i}, g_{i}, \mathcal{H}^{n}\right)\right\}$ converges to $\left(S^{n-p}(1) \times X, \mathfrak{m}\right)$ in the measured Gromov-Hausdorff topology. Put $M:=S^{n-p}(1) \times X$. Let $p_{1}: M \rightarrow S^{n-p}(1)$ and $p_{2}: M \rightarrow X$ be the projections. Define $f_{u}: M \rightarrow \mathbb{R}$ by $f_{u}(x):=p_{1}(x) \cdot u$ for each $u \in S^{n-p}(1) \subset \mathbb{R}^{n-p+1}$. Note that $(M, \mathfrak{m})$ satisfies the $\operatorname{RCD}^{*}(n-p-1, n)$ condition by [14, Theorem 3.22].

The goal of this section is to prove the following proposition.

Proposition 3.2. Under Assumption 3.1, there exists a Borel measure $\mathfrak{m}_{X}$ on $X$ such that $\mathfrak{m}=\underline{\mathcal{H}}^{n-p} \times \mathfrak{m}_{X}$ holds and $\left(X, \mathfrak{m}_{X}\right)$ satisfies the $\operatorname{RCD}(n-p-1, p)$ condition.

In this subsection, we show the splitting of the measure $\mathfrak{m}=\underline{\mathcal{H}}^{n-p} \times \mathfrak{m}_{X}$. Our approach has been inspired by [17]. We first show the following easy lemma.

Lemma 3.3. Define

$$
f_{k}: S^{n-p}(1) \times X \rightarrow \mathbb{R},\left(\left(u_{1}, \ldots, u_{n-p+1}\right), x\right) \mapsto u_{k}
$$

for each $k=1, \ldots, n-p+1$. Then, we have that $f_{k} \in \operatorname{TestF}(M)$ and the following properties:

(i) For each $k=1, \ldots, n-p+1$, the sequence $\left\{f_{k, i}\right\}$ converges to $f_{k}$ strongly in $L^{2}$ as $i \rightarrow \infty$.

(ii) For each $k=1, \ldots, n-p+1$, the sequence $\left\{\nabla f_{k, i}\right\}$ converges to $\nabla f_{k}$ strongly in $L^{2}$ as $i \rightarrow \infty$.

(iii) For each $k=1, \ldots, n-p+1$, we have that $\Delta f_{k}=-(n-p) f_{k}$.

(iv) For each $k, l=1, \ldots, n-p+1$ with $k \neq l$, we have that

$$
\begin{aligned}
& f_{k} f_{l}+\left\langle\nabla f_{k}, \nabla f_{l}\right\rangle=0, \\
& f_{k}^{2}+\left|\nabla f_{k}\right|^{2}=1
\end{aligned}
$$

m-a.e. in $M$. 
Proof. Clearly, $f_{k}$ is a Lipschitz function. If we get (iii), we have $\Delta f_{k}=-(n-p) f_{k} \in W^{1,2}(M)$, and so we have $f_{k} \in \operatorname{TestF}(M)$.

We first show that $\left\{f_{k, i}\right\}$ strongly converges to $f_{k}$ as $i \rightarrow \infty$ at each point $z \in M$. Note that by the gradient estimate for eigenfunctions [27, Theorem 7.3], there exists a constant $C>0$ such that $\left\|f_{k, i}\right\|_{L^{\infty}}+\left\|\nabla f_{k, i}\right\|_{L^{\infty}} \leq C$ holds for all $i \in \mathbb{Z}_{>0}$ and $k=1, \ldots, n-p+1$. Take arbitrary $z=(u, x) \in S^{n-p}(1) \times X$ and $z_{i} \in M_{i}\left(i \in \mathbb{Z}_{>0}\right)$ with $z_{i} \stackrel{\text { GH }}{\rightarrow} z$. Since we have $\Psi_{i}\left(z_{i}\right) \rightarrow u$ in $S^{n-p}(1)$, we have that

$$
\lim _{i \rightarrow \infty}\left|\frac{f_{k, i}\left(z_{i}\right)}{\left|\left(f_{1, i}, \ldots, f_{n-p+1, i}\right)\right|\left(z_{i}\right)}-f_{k}(z)\right|=0,
$$

and so $\lim _{i \rightarrow \infty}\left|f_{k, i}\left(z_{i}\right)-f_{k}(z)\right|=0$ by Proposition 2.18(iii). Since $f_{k, i}$ and $f_{k}$ are Lipschitz functions whose Lipschitz constants are bounded independently of $i$, we have that

$$
\begin{aligned}
& \frac{1}{\underline{\mathcal{H}}^{n}\left(B_{r}\left(z_{i}\right)\right)} \int_{B_{r}\left(z_{i}\right)}\left|f_{k, i}-\frac{1}{\mathfrak{m}\left(B_{r}(z)\right)} \int_{B_{r}(z)} f_{k} \mathrm{~d} \mathfrak{m}\right| \mathrm{d} \underline{\mathcal{H}}^{n} \leq \frac{1}{\underline{\mathcal{H}}^{n}\left(B_{r}\left(z_{i}\right)\right)} \\
& \quad \times \int_{B_{r}\left(z_{i}\right)}\left(\left|f_{k, i}-f_{k, i}\left(z_{i}\right)\right|+\left|f_{k, i}\left(z_{i}\right)-f_{k}(z)\right|+\frac{1}{\mathfrak{m}\left(B_{r}(z)\right)} \int_{B_{r}(z)}\left|f_{k}-f_{k}(z)\right| \mathrm{d} \mathfrak{m}\right) \mathrm{d} \underline{\mathcal{H}}^{n} \\
& \leq C r+\left|f_{k, i}\left(z_{i}\right)-f_{k}(z)\right| .
\end{aligned}
$$

Thus,

$$
\lim _{r \rightarrow 0} \limsup _{i \rightarrow \infty} \frac{1}{\underline{\mathcal{H}}^{n}\left(B_{r}\left(z_{i}\right)\right)} \int_{B_{r}\left(z_{i}\right)}\left|f_{k, i}-\frac{1}{\mathfrak{m}\left(B_{r}(z)\right)} \int_{B_{r}(z)} f_{k} \mathrm{~d} \mathfrak{m}\right| \mathrm{d} \underline{\mathcal{H}}^{n}=0 .
$$

Similarly, we have

$$
\lim _{r \rightarrow 0} \limsup _{i \rightarrow \infty} \frac{1}{\mathfrak{m}^{n}\left(B_{r}(z)\right)} \int_{B_{r}(z)}\left|f_{k}-\frac{1}{\underline{\mathcal{H}}^{n}\left(B_{r}\left(z_{i}\right)\right)} \int_{B_{r}\left(z_{i}\right)} f_{k, i} \mathrm{~d} \underline{\mathcal{H}}^{n}\right| \mathrm{d} \mathfrak{m}=0 .
$$

Therefore, we get $(i)$ by Proposition 2.16. We get $(i i)$ by Theorem 2.17(i). By Theorem 2.17(ii), we have

$$
\left\|\Delta f_{k}+(n-p) f_{k}\right\|_{L^{2}} \leq \liminf _{i \rightarrow \infty}\left\|\Delta f_{k, i}+(n-p) f_{k, i}\right\|_{L^{2}}=0
$$

and so we get $(i i i)$. For each $k=1, \ldots, n-p+1$, we have that

$$
\left\|f_{k}^{2}+\left|\nabla f_{k}\right|^{2}-1\right\|_{L^{1}}=\lim _{i \rightarrow \infty}\left\|f_{k, i}^{2}+\left|\nabla f_{k, i}\right|^{2}-1\right\|_{L^{1}},
$$

by [22, Propositions 3.11 and 3.45] and the original definition of the $L^{2}$ strong convergence [22, Definitions 3.25 and 3.58]. See also [5, Proposition 3.3 and Theorem 5.7]. By Proposition 2.18(i), we have $\lim _{i \rightarrow \infty}\left\|f_{k, i}^{2}+\left|\nabla f_{k, i}\right|^{2}-1\right\|_{L^{1}}=0$, and so we get $f_{k}^{2}+\left|\nabla f_{k}\right|^{2}=1 \mathfrak{m}$-a.e. in $M$. Similarly, applying Proposition $2.18(i)$ to $\left(f_{k, i} \pm f_{l, i}\right) / \sqrt{2}$, we get $f_{k} f_{l}+\left\langle\nabla f_{k}, \nabla f_{l}\right\rangle=0$ m-a.e. in $M$ for each $k, l=1, \ldots, n-p+1$ with $k \neq l$. These imply $(i v)$.

Let us apply Theorem 2.10 to vector fields generating rotations in $S^{n-p}(1)$.

Lemma 3.4. Take arbitrary $u=\left(u_{1}, \ldots, u_{n-p+1}\right), v=\left(v_{1}, \ldots, v_{n-p+1}\right) \in S^{n-p}(1)$ with $u \cdot v=0$ and $T>0$. Then, the vector field

$$
V_{u v}:=\sum_{i, j=1}^{n-p+1} u_{i} v_{j}\left(f_{i} \nabla f_{j}-f_{j} \nabla f_{i}\right)=f_{u} \nabla f_{v}-f_{v} \nabla f_{u}
$$


is an element of $\mathcal{D}(\operatorname{div})$ with $D^{\mathrm{sym}} V_{u v} \in L^{2}(M)$ and $\operatorname{div} V_{u v}=0$. Moreover, the regular Lagrangian flow $\mathrm{Fl}^{V_{u v}}: M \times[0, T] \rightarrow M$ for $V_{u v}$ exists and satisfies, for $\mathfrak{m}$-a.e. $z \in M$,

$$
\begin{aligned}
& f_{u}\left(\mathrm{Fl}_{t}^{V_{u v}}(z)\right)=f_{u}(z) \cos t-f_{v}(z) \sin t, \\
& f_{v}\left(\mathrm{Fl}_{t}^{V_{u v}}(z)\right)=f_{u}(z) \sin t+f_{v}(z) \cos t, \\
& f_{w}\left(\mathrm{Fl}_{t}^{V_{u v}}(z)\right)=f_{w}(z) \quad\left(w \in S^{n-p}(1) \text { with } u \cdot w=v \cdot w=0\right), \\
& p_{2}\left(\mathrm{Fl}_{t}^{V_{u v}}(z)\right)=p_{2}(z)
\end{aligned}
$$

for any $t \in[0, T]$. Moreover, $\mathrm{Fl}^{V_{u v}}$ preserves the measure $\mathfrak{m}$, i.e.,

$$
\left(\mathrm{Fl}_{t}^{V_{u v}}\right)_{*} \mathfrak{m}=\mathfrak{m}
$$

for any $t \in[0, T]$.

Proof. Since we have

$$
\operatorname{div}\left(f_{k} \nabla f_{l}\right)=-(n-p) f_{k} f_{l}+\left\langle\nabla f_{k}, \nabla f_{l}\right\rangle
$$

for each $k, l$ by (2.3.13) in [15], we get $\operatorname{div}\left(V_{u v}\right)=0$. We next check $D^{\operatorname{sym}} V_{u v} \in L^{2}(X)$. It is enough to show (2.1) when $f, g \in \operatorname{TestF}(M)$ because we have $\left\|\nabla f-\nabla P_{t} f\right\|_{L^{4}} \rightarrow 0$ and $\left\|\Delta f-\Delta P_{t} f\right\|_{L^{4}} \rightarrow 0$ as $t \rightarrow 0$ for each $f \in \mathcal{D}(\Delta)$ with $|\nabla f| \in L^{4}(M)$ and $\Delta f \in L^{4}(M)$. Note that

$$
L^{4}(T M):=\left\{V \in L^{2}(T M):|V| \in L^{4}(M)\right\}
$$

is a uniformly convex Banach space with the norm $\|V\|_{L^{4}}:=\||V|\|_{L^{4}}$, and that $\left\|\nabla P_{t} f\right\|_{L^{4}} \leq$ $\|\nabla f\|_{L^{4}}$ for each $f \in W^{1,2}(X)$ with $|\nabla f| \in L^{4}(X)$ by the $\operatorname{BL}(n-p-1, n)$ condition for $(M, \mathfrak{m})$. Combining these and $\nabla P_{t} f \rightarrow \nabla f$ in $L^{2}(T M)$, we get $\nabla P_{t} f \rightarrow \nabla f$ in $L^{4}(T M)$.

Take $f, g \in \operatorname{TestF}(M)$. A simple calculation implies

$$
\begin{aligned}
\int_{M}\left\langle V_{u v},\right. & \nabla f\rangle \Delta g+\left\langle V_{u v}, \nabla g\right\rangle \Delta f-\langle\nabla f, \nabla g\rangle \operatorname{div} V_{u v} \mathrm{dm} \\
= & \int_{M}\left\langle\nabla f_{v}, \nabla f\right\rangle \operatorname{div}\left(f_{u} \nabla g\right)+\left\langle\nabla f_{v}, \nabla g\right\rangle \operatorname{div}\left(f_{u} \nabla f\right)+f_{u}\left\langle\nabla f_{v}, \nabla\langle\nabla f, \nabla g\rangle\right\rangle \mathrm{d} \mathfrak{m} \\
& \quad-\int_{M}\left\langle\nabla f_{u}, \nabla f\right\rangle \operatorname{div}\left(f_{v} \nabla g\right)+\left\langle\nabla f_{u}, \nabla g\right\rangle \operatorname{div}\left(f_{v} \nabla f\right)+f_{v}\left\langle\nabla f_{u}, \nabla\langle\nabla f, \nabla g\rangle\right\rangle \mathrm{d} \mathfrak{m} .
\end{aligned}
$$

Note that we have $\langle\nabla f, \nabla g\rangle \in W^{1,2}(M)$ by [15, Proposition 3.1.3]. By [15, Definition 3.3.1 and Theorem 3.3.8], we get $D^{\mathrm{sym}} V_{u v} \in L^{2}(M)$. Thus, there exists a regular Lagrangian flow $\mathrm{Fl}^{V_{u v}}: M \times[0, T] \rightarrow M$ for $V_{u v}$ by Theorem 2.10.

For $\mathfrak{m}$-a.e. $z \in M$, we have

$$
\begin{aligned}
\frac{\mathrm{d}}{\mathrm{d} t} f_{w}\left(\mathrm{Fl}^{V_{u v}}(t, z)\right)= & \left(f_{u}\left\langle\nabla f_{v}, \nabla f_{w}\right\rangle-f_{v}\left\langle\nabla f_{u}, \nabla f_{w}\right\rangle\right)\left(\mathrm{Fl}^{V_{u v}}(t, z)\right) \\
= & \begin{cases}-f_{v}\left(\mathrm{Fl}^{V_{u v}}(t, z)\right) & (w=u), \\
f_{u}\left(\mathrm{Fl}^{V_{u v}}(t, z)\right) & (w=v), \\
0 & \left(w \in S^{n-p}(1) \text { with } u \cdot w=v \cdot w=0\right)\end{cases}
\end{aligned}
$$

for a.e. $t \in(0, T)$ by Lemma 3.3. This implies that for $\mathfrak{m}$-a.e. $z \in M$,

$$
f_{w}\left(\mathrm{Fl}^{V_{u v}}(t, z)\right)= \begin{cases}f_{u}(z) \cos t-f_{v}(z) \sin t & (w=u), \\ f_{u}(z) \sin t+f_{v}(z) \cos t & (w=v), \\ f_{w}(z) & \left(w \in S^{n-p}(1) \text { with } u \cdot w=v \cdot w=0\right)\end{cases}
$$

for any $t \in[0, T]$. 
A simple calculation implies that

$$
\left\langle\nabla f_{k}, \nabla\left(g \circ p_{2}\right)\right\rangle=0
$$

$\mathfrak{m}$-a.e. in $M$ for each $g \in \operatorname{LIP}(X)$ and $k=1, \ldots, n-p+1$ similarly to Lemma 3.11 (iv) below. Therefore, for each $g \in \operatorname{LIP}(X)$ and $\mathfrak{m}$-a.e. $z \in M$, we have

$$
\frac{\mathrm{d}}{\mathrm{d} t}\left(g \circ p_{2}\right)\left(\mathrm{Fl}^{V_{u v}}(t, z)\right)=0
$$

for a.e. $t \in[0, T]$, and so

$$
\left(g \circ p_{2}\right)\left(\mathrm{Fl}^{V_{u v}}(t, z)\right)=g \circ p_{2}(z)
$$

for any $t \in[0, T]$. Let $\left\{x_{j}\right\}_{j \in \mathbb{Z}_{>0}}$ be a countable dense subset of $X$. Then, by considering $g_{j}:=d\left(x_{j}, \cdot\right)$, we get that for $\mathfrak{m}$-a.e. $z \in M$,

$$
\mathrm{d}\left(x_{j}, p_{2}\left(\mathrm{Fl}^{V_{u v}}(t, z)\right)\right)=\mathrm{d}\left(x_{j}, p_{2}(z)\right)
$$

holds for any $j \in \mathbb{Z}_{>0}$ and $t \in[0, T]$. This implies for $\mathfrak{m}$-a.e. $z \in M, p_{2}\left(\mathrm{Fl}^{V_{u v}}(t, z)\right)=p_{2}(z)$ for any $t \in[0, T]$.

We have that $\mathrm{Fl}_{t}^{V_{u v}} \circ \mathrm{Fl}_{t}^{-V_{u v}}=\operatorname{Id}_{M}$ m-a.e. in $M$ for all $t \in[0, T]$ (note that $-V_{u v}=V_{v u}$ ), and so

$$
\mathfrak{m}=\left(\mathrm{Fl}_{t}^{V_{u v}}\right)_{*}\left(\mathrm{Fl}_{t}^{-V_{u v}}\right)_{*} \mathfrak{m} \leq\left(\mathrm{Fl}_{t}^{V_{u v}}\right)_{*} \mathfrak{m} \leq \mathfrak{m} .
$$

This implies the final assertion.

Corollary 3.5. For any $T \in \mathrm{SO}(n-p+1)$, the transformation

$$
T: S^{n-p}(1) \times X \rightarrow S^{n-p}(1) \times X,(u, x) \mapsto(T u, x)
$$

preserves the measure $\mathfrak{m}$.

Proof. Modifying on m-negligible subset, we have that $\mathrm{Fl}_{t}^{V_{u v}} \in \mathrm{SO}(n-p+1)$ for each $u, v \in$ $S^{n-p}(1)$ with $u \cdot v=0$ and $t \in[0,2 \pi]$. Conversely, any $T \in \mathrm{SO}(n-p+1)$ can be expressed as a composition of several transformations of the form $\mathrm{Fl}_{t}^{V_{u v}}$. Thus, we get the corollary.

The following proposition is the goal of this subsection.

Proposition 3.6. Define a Borel measure $\mathfrak{m}_{X}$ on $X$ by

$$
\mathfrak{m}_{X}:=\left(p_{2}\right)_{*} \mathfrak{m} .
$$

Then, we have $\mathfrak{m}=\underline{\mathcal{H}}^{n-p} \times \mathfrak{m}_{X}$.

Proof. We first fix a Borel subset $B \subset X$. Define a Borel measure $\mu_{B}$ on $S^{n-p}(1)$ by

$$
\mu_{B}:=\left(p_{1}\right)_{*}\left(\left.\mathfrak{m}\right|_{p_{2}^{-1}(B)}\right),
$$

i.e., we define

$$
\mu_{B}(A):=\mathfrak{m}(A \times B)
$$

for any subset $A \subset S^{n-p}(1)$.

Claim 3.7. Each $T \in \mathrm{SO}(n-p+1)$ preserves the measure $\mu_{B}$. 
Proof. We immediately have the claim by Corollary 3.5 .

Claim 3.8. $\mu_{B} \ll \mathcal{H}^{n-p}$.

Proof. By the volume estimate relative to $\mathcal{H}^{n-p}$ on $S^{n-p}(1)$, there exists a constant $C>0$ such that

$$
\max \left\{k \in \mathbb{Z}_{>0}: \begin{array}{l}
\text { there exist } x_{1}, \ldots, x_{k} \in S^{n-p}(1) \text { such that } \\
B_{r}\left(x_{i}\right) \cap B_{r}\left(x_{j}\right)=\varnothing \text { holds for each } i \neq j
\end{array}\right\} \geq r^{-(n-p)} / C
$$

holds for all $r>0$.

Take $r>0$. We can choose $k \in \mathbb{Z}_{>0}$ with $k \geq r^{-(n-p)} / C$ and $x_{1}, \ldots, x_{k} \in S^{n-p}(1)$ such that $B_{r}\left(x_{i}\right) \cap B_{r}\left(x_{j}\right)=\varnothing$ holds for each $i \neq j$. By Claim 3.7, we have that

$$
\mu_{B}\left(B_{r}\left(x_{i}\right)\right)=\mu_{B}\left(B_{r}\left(x_{j}\right)\right)=\mu_{B}\left(B_{r}(x)\right)
$$

for all $i, j=1, \ldots, k$ and $x \in S^{n-p}(1)$. Therefore, we get that $\mu_{B}\left(B_{r}(x)\right) \leq C r^{n-p} \mu_{B}\left(S^{n-p}(1)\right)$ for all $x \in S^{n-p}(1)$.

Take arbitrary subset $A \subset S^{n-p}(1)$ with $\mathcal{H}^{n-p}(A)=0$ and $\epsilon>0$. Then, by the definition of the Hausdorff measure, there exists a sequence of subsets $\left\{S_{j}\right\}_{j \in \mathbb{Z}_{>0}}$ of $S^{n-p}(1)$ such that $A \subset \bigcup_{j=1}^{\infty} S_{j}$ and

$$
\sum_{j=1}^{\infty}\left(\operatorname{diam} S_{j}\right)^{n-p}<\epsilon
$$

Choose $x_{j} \in S_{j}$ for each $j$. Then, we have $S_{j} \subset B_{\operatorname{diam} S_{j}}\left(x_{j}\right)$, and so

$$
\mu_{B}(A) \leq \sum_{j=1}^{\infty} \mu_{B}\left(B_{\operatorname{diam} S_{j}}\left(x_{j}\right)\right) \leq C \sum_{j=1}^{\infty}\left(\operatorname{diam} S_{j}\right)^{n-p} \mu_{B}\left(S^{n-p}(1)\right) \leq C \epsilon \mu_{B}\left(S^{n-p}(1)\right) .
$$

Letting $\epsilon \rightarrow 0$, we obtain $\mu_{B}(A)=0$ and get the claim.

By Claim 3.8 and the Radon-Nikodym theorem, we have the representation $\mu_{B}=\rho \mathcal{H}^{n-p}$, where $\rho: S^{n-p}(1) \rightarrow[0, \infty]$ is some Borel function. By Claim 3.7, we have that for each $T \in$ $\mathrm{SO}(n-p+1)$

$$
\rho \circ T=\rho
$$

$\mathcal{H}^{n-p}$-a.e. in $S^{n-p}(1)$. This implies that $\rho$ is constant $\mathcal{H}^{n-p}$-a.e. in $S^{n-p}(1)$. We have that

$$
\rho \mathcal{H}^{n-p}\left(S^{n-p}(1)\right)=\mu_{B}\left(S^{n-p}(1)\right)=\mathfrak{m}\left(S^{n-p}(1) \times B\right)=\mathfrak{m}_{X}(B),
$$

and so $\mu_{B}=\mathfrak{m}_{X}(B) \underline{\mathcal{H}}^{n-p}$.

For each Borel sets $A \subset S^{n-p}(1)$ and $B \subset X$, we get that

$$
\mathfrak{m}(A \times B)=\mu_{B}(A)=\underline{\mathcal{H}}^{n-p}(A) \mathfrak{m}_{X}(B) .
$$

This implies the proposition. 


\subsection{Product metric measure spaces and the $\mathrm{RCD}^{*}$ condition}

In the previous subsection we showed that there exists a Borel measure $\mathfrak{m}_{X}$ on $\mathrm{X}$ such that $\mathfrak{m}=\underline{\mathcal{H}}^{n-p} \times \mathfrak{m}_{X}$ holds under Assumption 3.1. In this subsection we show that $\left(X, \mathfrak{m}_{X}\right)$ satisfies the $\operatorname{RCD}(n-p-1, p)$ condition.

More generally, we consider the following assumption.

Assumption 3.9. Let $K, N \in \mathbb{R}$ with $N \geq 1$ and $\left(X_{i}, d_{i}, \mathfrak{m}_{i}\right)(i=1,2)$ be compact metric measure spaces. Put $(M, d, \mathfrak{m}):=\left(X_{1} \times X_{2}, d_{1} \times d_{2}, \mathfrak{m}_{1} \times \mathfrak{m}_{2}\right)$. Moreover, we assume the following:

- $\mathfrak{m}_{i}\left(X_{i}\right)=1(i=1,2)$,

- $(M, d, \mathfrak{m})$ satisfies the $\operatorname{RCD}^{*}(K, N)$ condition.

For each $i=1,2$, let $p_{i}: M \rightarrow X_{i}$ denote the projection.

The goal of this subsection is to prove the following proposition:

Proposition 3.10. In addition to Assumption 3.9, we assume that $\left(X_{1}, d_{1}, \mathfrak{m}_{1}\right)$ is an $n$-dimensional closed Riemannian manifold with the Riemannian distance and $\mathfrak{m}_{1}=\underline{\mathcal{H}}^{n}$. Then, $\left(X_{2}, d_{2}, \mathfrak{m}_{2}\right)$ satisfies the $\operatorname{RCD}^{*}(K, N-n)$ condition if $N-n \geq 1$.

Note that if $\left(Y_{i}, d_{i}^{\prime}, \mathfrak{m}_{i}^{\prime}\right)(i=1,2)$ are $\operatorname{RCD}^{*}\left(K, N_{i}\right)$ spaces, then the product space $\left(Y_{1} \times Y_{2}\right.$, $\left.d_{1}^{\prime} \times d_{2}^{\prime}, \mathfrak{m}_{1}^{\prime} \times \mathfrak{m}_{2}^{\prime}\right)$ satisfies the $\operatorname{RCD}^{*}\left(K, N_{1}+N_{2}\right)$ condition by [14, Theorem 3.23].

We first show the following easy lemma.

Lemma 3.11. Under Assumption 3.9, we have the following properties:

(i) For any $f \in \operatorname{LIP}(M)$ and $x=\left(x_{1}, x_{2}\right) \in M$, we have that

$$
\operatorname{Lip}_{M}(f)(x) \geq\left(\operatorname{Lip}_{X_{1}}\left(f\left(\cdot, x_{2}\right)\right)\right)\left(x_{1}\right)
$$

and that

$$
\operatorname{Lip}_{M}(f)(x) \geq\left(\operatorname{Lip}_{X_{2}}\left(f\left(x_{1}, \cdot\right)\right)\right)\left(x_{2}\right) .
$$

(ii) For any $f \in \operatorname{LIP}\left(X_{i}\right)(i=1,2)$, we have that

$$
\left(\operatorname{Lip}_{X_{i}} f\right) \circ p_{i}=\operatorname{Lip}_{M}\left(f \circ p_{i}\right) .
$$

(iii) For each $i=1,2$, the map $p_{i}^{*}: L^{2}\left(X_{i}, \mathfrak{m}_{i}\right) \rightarrow L^{2}(M, \mathfrak{m}), f \mapsto f \circ p_{i}$ induces an isometric immersion $p_{i}^{*}: W^{1,2}\left(X_{i}\right) \rightarrow W^{1,2}(M)$, and we have that

$$
\left|\nabla\left(f \circ p_{i}\right)\right|=|\nabla f| \circ p_{i}
$$

$\mathfrak{m}$-a.e. in $M$ for any $f \in W^{1,2}\left(X_{i}\right)(i=1,2)$.

(iv) For any $f \in W^{1,2}\left(X_{1}\right)$ and $h \in \operatorname{LIP}(M)$, we have that

$$
\left\langle\nabla\left(f \circ p_{1}\right), \nabla h\right\rangle(x)=\left\langle\nabla f, \nabla\left(h\left(\cdot, x_{2}\right)\right)\right\rangle\left(x_{1}\right)
$$

for $\mathfrak{m}$-a.e. $x=\left(x_{1}, x_{2}\right) \in M$. The similar result holds for the element of $W^{1,2}\left(X_{2}\right)$.

(v) For any $f_{i} \in W^{1,2}\left(X_{i}\right)(i=1,2)$, we have that

$$
\left\langle\nabla\left(f_{1} \circ p_{1}\right), \nabla\left(f_{2} \circ p_{2}\right)\right\rangle=0
$$

m-a.e. in $M$. 
Proof. We get $(i)$ and $(i i)$ straightforward by the definition.

We show (iii) for $i=1$. Take arbitrary $f \in W^{1,2}\left(X_{1}\right)$. For any sequence $\left\{f_{n}\right\} \subset \operatorname{LIP}\left(X_{1}\right)$ with $\lim _{n \rightarrow \infty}\left\|f_{n}-f\right\|_{L^{2}}=0$, we have $\lim _{n \rightarrow \infty}\left\|f_{n} \circ p_{1}-f \circ p_{1}\right\|_{L^{2}}=0$, and so

$$
\mathrm{Ch}_{M}\left(f \circ p_{1}\right) \leq \frac{1}{2} \liminf _{n \rightarrow \infty} \int_{M}\left(\operatorname{Lip}_{M}\left(f_{n} \circ p_{1}\right)\right)^{2} \mathrm{dm}=\frac{1}{2} \liminf _{n \rightarrow \infty} \int_{X_{1}}\left(\operatorname{Lip}_{X_{1}} f_{n}\right)^{2} \mathrm{dm} \mathfrak{m}_{1}
$$

by (ii). This implies $\mathrm{Ch}_{M}\left(f \circ p_{1}\right) \leq \mathrm{Ch}_{X_{1}}(f)$ and $f \circ p_{1} \in W^{1,2}(M)$.

We next show $\mathrm{Ch}_{M}\left(f \circ p_{1}\right) \geq \mathrm{Ch}_{X_{1}}(f)$. Take any sequence $\left\{f_{n}\right\} \subset \operatorname{LIP}(M)$ with $\epsilon_{n}:=$ $\left\|f_{n}-f \circ p_{1}\right\|_{L^{2}}^{2} \rightarrow 0$ as $n \rightarrow \infty$. We can assume $\epsilon_{n}<1$ for each $n$. We have that

$$
\begin{aligned}
& \epsilon_{n}=\int_{X_{2}} \int_{X_{1}}\left(f_{n}\left(x_{1}, x_{2}\right)-f\left(x_{1}\right)\right)^{2} \mathrm{~d} \mathfrak{m}_{1}\left(x_{1}\right) \mathrm{d} \mathfrak{m}_{2}\left(x_{2}\right), \\
& \frac{1}{2} \int_{M}\left(\operatorname{Lip}_{M} f_{n}\right)^{2} \mathrm{~d} \mathfrak{m} \geq \frac{1}{2} \int_{X_{2}} \int_{X_{1}}\left(\operatorname{Lip}_{X_{1}} f_{n}\left(\cdot, x_{2}\right)\right)^{2}\left(x_{1}\right) \mathrm{dm}_{1}\left(x_{1}\right) \mathrm{d} \mathfrak{m}_{2}\left(x_{2}\right)
\end{aligned}
$$

by $(i)$, and so

$$
\begin{aligned}
& \mathfrak{m}_{2}\left(\left\{x_{2} \in X_{2}: \int_{X_{1}}\left(f_{n}\left(\cdot, x_{2}\right)-f\right)^{2} \mathrm{~d} \mathfrak{m}_{1}>2 \epsilon_{n}^{1 / 2}\right\}\right) \leq \frac{1}{2} \epsilon_{n}^{1 / 2} \\
& \mathfrak{m}_{2}\left(\left\{x_{2} \in X_{2}: \int_{X_{1}}\left(\operatorname{Lip}_{X_{1}}\left(f_{n}\left(\cdot, x_{2}\right)\right)^{2} \mathrm{dm}_{1}>\left(1+\epsilon_{n}^{1 / 2}\right) \int_{M}\left(\operatorname{Lip}_{M} f_{n}\right)^{2} \mathrm{~d} \mathfrak{m}\right\}\right) \leq \frac{1}{1+\epsilon^{1 / 2}}\right.
\end{aligned}
$$

Since we have

$$
\frac{1}{2} \epsilon_{n}^{1 / 2}+\frac{1}{1+\epsilon_{n}^{1 / 2}}<1,
$$

we can take a sequence $\left\{x_{2}(n)\right\} \subset X_{2}$ such that

$$
\begin{aligned}
& \int_{X_{1}}\left(f_{n}\left(\cdot, x_{2}(n)\right)-f\right)^{2} \mathrm{~d} \mathfrak{m}_{1} \leq 2 \epsilon_{n}^{1 / 2}, \\
& \int_{X_{1}}\left(\operatorname{Lip}_{X_{1}}\left(f_{n}\left(\cdot, x_{2}(n)\right)\right)\right)^{2} \mathrm{~d} \mathfrak{m}_{1} \leq\left(1+\epsilon_{n}^{1 / 2}\right) \int_{M}\left(\operatorname{Lip}_{M} f_{n}\right)^{2} \mathrm{~d} \mathfrak{m}
\end{aligned}
$$

for each $n$. Put $g_{n}:=f_{n}\left(\cdot, x_{2}(n)\right) \in \operatorname{LIP}\left(X_{1}\right)$. Then, we have $\left\|g_{n}-f\right\|_{L^{2}} \rightarrow 0$ as $n \rightarrow \infty$ and

$$
\mathrm{Ch}_{X_{1}}(f) \leq \frac{1}{2} \liminf _{n \rightarrow \infty} \int_{X_{1}}\left(\operatorname{Lip}_{X_{1}} g_{n}\right)^{2} \mathrm{dm} \mathfrak{m}_{1} \leq \frac{1}{2} \liminf _{n \rightarrow \infty} \int_{M}\left(\operatorname{Lip}_{M} f_{n}\right)^{2} \mathrm{dm} .
$$

Thus, we get $\mathrm{Ch}_{M}\left(f \circ p_{1}\right) \geq \mathrm{Ch}_{X_{1}}(f)$, and so $\mathrm{Ch}_{M}\left(f \circ p_{1}\right)=\mathrm{Ch}_{X_{1}}(f)$. Therefore, $p_{1}^{*}: W^{1,2}\left(X_{1}\right) \rightarrow$ $W^{1,2}(M)$ is isometric

Let us show that $\left|\nabla\left(f \circ p_{1}\right)\right|=|\nabla f| \circ p_{1}$ m-a.e. in $M$. Take $f_{n} \in \operatorname{LIP}\left(X_{1}\right)$ such that $f_{n} \rightarrow f$ and $\operatorname{Lip}_{X_{1}} f_{n} \rightarrow|\nabla f|$ in $L^{2}$. Then, we have $f_{n} \circ p_{1} \rightarrow f \circ p_{1}, \operatorname{Lip}_{M}\left(f_{n} \circ p_{1}\right)=\left(\operatorname{Lip}_{X_{1}} f_{n}\right) \circ p_{1} \rightarrow|\nabla f| \circ p_{1}$ in $L^{2}$ and

$$
\mathrm{Ch}_{M}\left(f \circ p_{1}\right)=\mathrm{Ch}_{X_{1}}(f)=\frac{1}{2} \int_{M}\left(|\nabla f| \circ p_{1}\right)^{2} \mathrm{dm} .
$$

This implies $\left|\nabla\left(f \circ p_{1}\right)\right|=|\nabla f| \circ p_{1} \mathfrak{m}$-a.e. in $M$.

Let us prove $(i v)$. We first consider the case $f \in \operatorname{Lip}\left(X_{1}\right)$. Then, we have

$$
\begin{aligned}
\left\langle\nabla\left(f \circ p_{1}\right), \nabla h\right\rangle\left(x_{1}, x_{2}\right) & =\lim _{\epsilon \rightarrow 0} \frac{1}{2 \epsilon}\left(\operatorname{Lip}_{M}\left(f \circ p_{1}+\epsilon h\right)^{2}-\operatorname{Lip}_{M}\left(f \circ p_{1}\right)^{2}\right)\left(x_{1}, x_{2}\right) \\
& \geq \lim _{\epsilon \rightarrow 0} \frac{1}{2 \epsilon}\left(\operatorname{Lip}_{X_{1}}\left(f+\epsilon h\left(\cdot, x_{2}\right)\right)^{2}-\operatorname{Lip}_{X_{1}}(f)^{2}\right)\left(x_{1}\right) \\
& =\left\langle\nabla f, \nabla\left(h\left(\cdot, x_{2}\right)\right)\right\rangle\left(x_{1}\right)
\end{aligned}
$$


for m-a.e. $\left(x_{1}, x_{2}\right) \in M$. By considering $-h$ instead of $h$, we also get

$$
\left\langle\nabla\left(f \circ p_{1}\right), \nabla h\right\rangle\left(x_{1}, x_{2}\right) \leq\left\langle\nabla f, \nabla\left(h\left(\cdot, x_{2}\right)\right)\right\rangle\left(x_{1}\right),
$$

and so

$$
\left\langle\nabla\left(f \circ p_{1}\right), \nabla h\right\rangle\left(x_{1}, x_{2}\right)=\left\langle\nabla f, \nabla\left(h\left(\cdot, x_{2}\right)\right)\right\rangle\left(x_{1}\right)
$$

for m-a.e. $\left(x_{1}, x_{2}\right) \in M$. For general $f \in W^{1,2}\left(X_{1}\right)$, approximating $f$ by Lipschitz functions, we get $(i v)$.

Finally we show $(v)$. We have $(v)$ for each $f_{i} \in \operatorname{LIP}\left(X_{i}\right)(i=1,2)$ by $(i v)$. For general $f_{i} \in W^{1,2}\left(X_{i}\right)$, approximating $f_{i}$ by Lipschitz functions, we get $(v)$.

We immediately get the following corollary by Lemma 3.11(iii).

Corollary 3.12. Under Assumption 3.9, we have that the metric measure space $\left(X_{i}, d_{i}, m_{i}\right)$ is infinitesimally Hilbertian and satisfies the Sobolev-to-Lipschitz property for each $i=1,2$.

For any $f_{i} \in L^{2}\left(X_{i}\right)$, we shall denote $f_{i} \circ p_{i} \in L^{2}(M)$ by $f_{i}$ briefly if there is no confusion.

Lemma 3.13. Under Assumption 3.9, we have $f_{1} f_{2} \in W^{1,2}(M)$ and

$$
\nabla\left(f_{1} f_{2}\right)=f_{1} \nabla f_{2}+f_{2} \nabla f_{1} \in L^{2}(T M)
$$

for any $f_{i} \in W^{1,2}\left(X_{i}\right)(i=1,2)$.

Proof. Take sequences $\left\{f_{i, n}\right\}_{n \in \mathbb{Z}_{>0}} \subset \operatorname{LIP}\left(X_{i}\right)(i=1,2)$ such that $f_{i, n} \rightarrow f_{i}$ and $\operatorname{Lip}_{X_{i}}\left(f_{i, n}\right) \rightarrow$ $\left|\nabla f_{i, n}\right|$ in $L^{2}\left(X_{i}\right)$ as $n \rightarrow \infty$. We have $f_{1, n} f_{2, n} \in W^{1,2}(M)$ and $\nabla\left(f_{1, n} f_{2, n}\right)=f_{1, n} \nabla f_{2, n}+$ $f_{2, n} \nabla f_{1, n}$ by [15, Theorem 2.2.6]. Then, $f_{1, n} f_{2, n} \rightarrow f_{1} f_{2}$ in $L^{2}(M)$ and

$$
\nabla\left(f_{1, n} f_{2, n}\right)=f_{1, n} \nabla f_{2, n}+f_{2, n} \nabla f_{1, n} \rightarrow f_{1} \nabla f_{2}+f_{2} \nabla f_{1}
$$

in $L^{2}(T M)$. Thus, we get that $f_{1, n} f_{2, n} \rightarrow f_{1} f_{2}$ in $W^{1,2}(M)$ and $\nabla\left(f_{1} f_{2}\right)=f_{1} \nabla f_{2}+f_{2} \nabla f_{1} \in$ $L^{2}(T M)$.

Let us consider the Laplacian on $M$.

Lemma 3.14. Under Assumption 3.9, we have the following properties:

(i) For each $i=1,2$, the map $p_{i}^{*}: L^{2}\left(X_{i}\right) \rightarrow L^{2}(M)$ induces a map $p_{i}^{*}: \mathcal{D}\left(\Delta_{X_{i}}\right) \rightarrow \mathcal{D}\left(\Delta_{M}\right)$, and we have that

$$
\left(\Delta_{X_{i}} f\right) \circ p_{i}=\Delta_{M}\left(f \circ p_{i}\right)
$$

for any $f \in \mathcal{D}\left(\Delta_{X_{i}}\right)(i=1,2)$. Thus, we use the same notation $\Delta$ for $\Delta_{M}$ and $\Delta_{X_{i}}$ $(i=1,2)$. For any $f \in \mathcal{D}\left(\Delta_{X_{i}}\right)$, we shall denote $\left(\Delta_{X_{i}} f\right) \circ p_{i}$ by $\Delta f$ briefly if there is no confusion.

(ii) For any $f_{i} \in \mathcal{D}\left(\Delta_{X_{i}}\right)(i=1,2)$, we have that $f_{1} f_{2} \in \mathcal{D}\left(\Delta_{M}\right)$ and that

$$
\Delta\left(f_{1} f_{2}\right)=f_{1} \Delta f_{2}+\left(\Delta f_{1}\right) f_{2}
$$


Proof. We show $(i)$ for $i=1$. Take arbitrary $f \in \mathcal{D}\left(\Delta_{X_{1}}\right)$. Then, for any $\phi \in \operatorname{LIP}(M)$, we have

$$
\begin{aligned}
\int_{M}\left\langle\nabla\left(f \circ p_{1}\right), \nabla \phi\right\rangle & =\int_{X_{2}} \int_{X_{1}}\left\langle\nabla f, \nabla\left(\phi\left(\cdot, x_{2}\right)\right)\right\rangle\left(x_{1}\right) \mathrm{dm}_{1}\left(x_{1}\right) \mathrm{d} \mathfrak{m}_{2}\left(x_{2}\right) \\
& =-\int_{X_{2}} \int_{X_{1}} \Delta_{X_{1}} f\left(x_{1}\right) \phi\left(x_{1}, x_{2}\right) \mathrm{dm}_{1}\left(x_{1}\right) \mathrm{d} \mathfrak{m}_{2}\left(x_{2}\right) \\
& =-\int_{M}\left(\Delta_{X_{1}} f\right) \circ p_{1} \cdot \phi \mathrm{d} \mathfrak{m} .
\end{aligned}
$$

Since $\operatorname{LIP}(M) \subset W^{1,2}(M)$ is dense with respect to the norm $\|\cdot\|_{W^{1,2}}$, we get $(i)$.

We next show $(i i)$. Take arbitrary $f_{i} \in \mathcal{D}\left(\Delta_{X_{i}}\right)(i=1,2)$. Then, for any $\phi \in \operatorname{LIP}(M)$, we have

$$
\begin{aligned}
\int_{M}\left\langle\nabla\left(f_{1} f_{2}\right), \nabla \phi\right\rangle= & \int_{X_{1}} \int_{X_{2}} f_{1}\left(x_{1}\right)\left\langle\nabla f_{2}, \nabla\left(\phi\left(x_{1}, \cdot\right)\right)\right\rangle\left(x_{2}\right) \mathrm{d} \mathfrak{m}_{1}\left(x_{1}\right) \mathrm{d} \mathfrak{m}_{2}\left(x_{2}\right) \\
& +\int_{X_{1}} \int_{X_{2}} f_{2}\left(x_{2}\right)\left\langle\nabla f_{1}, \nabla\left(\phi\left(\cdot, x_{2}\right)\right)\right\rangle\left(x_{1}\right) \mathrm{d} \mathfrak{m}_{1}\left(x_{1}\right) \mathrm{d} \mathfrak{m}_{2}\left(x_{2}\right) \\
= & -\int_{M}\left(f_{1} \Delta f_{2}+\left(\Delta f_{1}\right) f_{2}\right) \phi \mathrm{d} \mathfrak{m} .
\end{aligned}
$$

Since $\operatorname{LIP}(M) \subset W^{1,2}(M)$ is dense, we get $(i i)$.

Our goal is to show $\left(X_{2}, d_{2}, \mathfrak{m}_{2}\right)$ satisfies the $\operatorname{RCD}^{*}(K, N-n)$ condition under the assumption of Proposition 3.10. However, we can show the following weaker assertion under Assumption 3.9.

Corollary 3.15. Under Assumption 3.9, we have that the metric measure space $\left(X_{i}, d_{i}, \mathfrak{m}_{i}\right)$ satisfies the $\operatorname{RCD}^{*}(K, N)$ condition for each $i=1,2$.

Proof. We only need to show that $\left(X_{i}, d_{i}, \mathfrak{m}_{i}\right)$ satisfies the $\operatorname{BE}(K, N)$ condition by Corollary 3.12. For any $u_{i} \in \mathcal{D}\left(\Delta_{X_{i}}\right)$ with $\Delta u_{i} \in W^{1,2}\left(X_{i}\right)$ and $\phi_{i} \in \mathcal{D}\left(\Delta_{X_{i}}\right) \cap L^{\infty}\left(X_{i}\right)$ with $\phi_{i} \geq 0$ and $\Delta_{X_{i}} \phi_{i} \in L^{\infty}\left(X_{i}\right)$, applying the $\operatorname{BE}(K, N)$ condition for $(M, d, \mathfrak{m})$ to $u_{i} \circ p_{i}, \phi_{i} \circ p_{i} \in \mathcal{D}\left(\Delta_{M}\right)$, we get the $\operatorname{BE}(K, N)$ condition for $\left(X_{i}, d_{i}, \mathfrak{m}_{i}\right)$.

The following proposition is crucial to show Proposition 3.10. We show the $\operatorname{BE}(K, N-n)$ condition with an error term.

Proposition 3.16. In addition to Assumption 3.9, we assume that $n$ is an integer with $N-n \geq 1$ and that $\left(X_{1}, d_{1}, \mathfrak{m}_{1}\right)$ is an $n$-dimensional closed Riemannian manifold with the Riemannian distance and $\mathfrak{m}_{1}=\underline{\mathcal{H}}^{n}$. Then, for all $u \in \mathcal{D}\left(\Delta_{X_{2}}\right)$ with $\Delta u \in W^{1,2}\left(X_{2}\right)$ and all $\phi \in \mathcal{D}\left(\Delta_{X_{2}}\right) \cap$ $L^{\infty}\left(X_{2}\right)$ with $\phi \geq 0$ and $\Delta \phi \in L^{\infty}\left(X_{2}\right)$, we have

$$
\begin{aligned}
& \frac{1}{2} \int_{X_{2}} \Delta \phi|\nabla u|^{2} \mathrm{dm}_{2} \\
& \quad \geq \int_{X_{2}} \phi\left(\langle\nabla \Delta u, \nabla u\rangle+K|\nabla u|^{2}+\frac{(\Delta u)^{2}}{N-n}-\frac{2 n}{N(N-n)}(\Delta u-2(N-n) u)^{2}\right) \mathrm{d} \mathfrak{m}_{2} .
\end{aligned}
$$

Proof. Take $\psi \in C^{\infty}(\mathbb{R})$ such that

$$
\psi(t)= \begin{cases}1, & |t| \leq \frac{1}{2} \\ 0, & |t| \geq 1\end{cases}
$$


and $\psi \geq 0$. Fix $p \in X_{1}$. Take sufficiently small $\epsilon>0$ so that we can take $\psi_{\epsilon}$ and $f_{\epsilon}$ below as smooth functions. Define $\psi_{\epsilon} \in C^{\infty}\left(X_{1}\right)$ by

$$
\psi_{\epsilon}\left(x_{1}\right):=\psi\left(\frac{d\left(p, x_{1}\right)}{\epsilon}\right)
$$

for each $x_{1} \in X_{1}$, and take $f_{\epsilon} \in C^{\infty}\left(X_{1}\right)$ such that

$$
f_{\epsilon}\left(x_{1}\right)= \begin{cases}1+d_{1}\left(p, x_{1}\right)^{2}, & d\left(p, x_{1}\right) \leq \epsilon, \\ 0, & d\left(p, x_{1}\right) \geq 2 \epsilon .\end{cases}
$$

Then, there exists a constant $C>0$ such that

$$
\begin{aligned}
& \left|\operatorname{Hess} f_{\epsilon}-\frac{\Delta f_{\epsilon}}{n} g_{X_{1}}\right|\left(x_{1}\right) \leq C d\left(p, x_{1}\right), \\
& \left|\Delta f_{\epsilon}-2 n\right|\left(x_{1}\right) \leq C d\left(p, x_{1}\right), \\
& \left|\nabla f_{\epsilon}\right|\left(x_{1}\right) \leq C d\left(p, x_{1}\right), \\
& \left|f_{\epsilon}-1\right|\left(x_{1}\right) \leq C d\left(p, x_{1}\right)
\end{aligned}
$$

for all $x_{1} \in B_{\epsilon}(p)$. Note that we can take such a constant independently of $\epsilon$.

Claim 3.17. There exists a constant $C>0$ such that for all sufficiently small $\epsilon>0$, all $u \in \mathcal{D}\left(\Delta_{X_{2}}\right)$ with $\Delta u \in W^{1,2}\left(X_{2}\right)$ and all $\phi \in \mathcal{D}\left(\Delta_{X_{2}}\right) \cap L^{\infty}\left(X_{2}\right)$ with $\phi \geq 0$ and $\Delta \phi \in L^{\infty}\left(X_{2}\right)$, we have

$$
\begin{aligned}
\frac{1}{2} \int_{X_{2}} \Delta \phi|\nabla u|^{2} \mathrm{~d} \mathfrak{m}_{2} \geq & \int_{X_{2}} \phi\left(\langle\nabla \Delta u, \nabla u\rangle+K|\nabla u|^{2}+\frac{(\Delta u)^{2}}{N-n}\right) \mathrm{d} \mathfrak{m}_{2} \\
& -C \epsilon^{2} \int_{X_{2}} \phi\left(u^{2}+|\nabla u|^{2}+(\Delta u)^{2}\right) \mathrm{d} \mathfrak{m}_{2} \\
& -\frac{2 n}{N(N-n)} \int_{X_{2}} \phi(\Delta u-2(N-n) u)^{2} \mathrm{~d} \mathfrak{m}_{2} .
\end{aligned}
$$

Proof. We have that $f_{\epsilon} u \in \mathcal{D}\left(\Delta_{M}\right)$ with $\Delta\left(f_{\epsilon} u\right)=f_{\epsilon} \Delta u+\left(\Delta f_{\epsilon}\right) u \in W^{1,2}(M)$ and that $\psi_{\epsilon} \phi \in \mathcal{D}\left(\Delta_{M}\right) \cap L^{\infty}(M)$ with $\psi_{\epsilon} \phi \geq 0$ and $\Delta\left(\psi_{\epsilon} \phi\right)=\psi_{\epsilon} \Delta \phi+\left(\Delta \psi_{\epsilon}\right) \phi \in L^{\infty}(M)$ by Lemma 3.13 and Lemma 3.14. Thus, we can apply the $\operatorname{BE}(K, N)$ condition to the pair $\left(f_{\epsilon} u, \psi_{\epsilon} \phi\right)$ and get

$$
\begin{aligned}
\frac{1}{2} \int_{M} \Delta\left(\psi_{\epsilon} \phi\right)\left|\nabla\left(f_{\epsilon} u\right)\right|^{2} \mathrm{~d} \mathfrak{m} \geq & \int_{M}\left(\psi_{\epsilon} \phi\right)\left\langle\nabla \Delta\left(f_{\epsilon} u\right), \nabla\left(f_{\epsilon} u\right)\right\rangle \mathrm{d} \mathfrak{m} \\
& +K \int_{M} \psi_{\epsilon} \phi\left|\nabla\left(f_{\epsilon} u\right)\right|^{2} \mathrm{~d} \mathfrak{m}+\frac{1}{N} \int_{M} \psi_{\epsilon} \phi\left(\Delta\left(f_{\epsilon} u\right)\right)^{2} \mathrm{~d} \mathfrak{m} .
\end{aligned}
$$

We calculate each terms.

We have

$$
\begin{aligned}
\frac{1}{2} \int_{M} \Delta & \left(\psi_{\epsilon} \phi\right)\left|\nabla\left(f_{\epsilon} u\right)\right|^{2} \mathrm{~d} \mathfrak{m} \\
= & \int_{X_{1}} \psi_{\epsilon}\left(\left\langle\nabla \Delta f_{\epsilon} \nabla f_{\epsilon}\right\rangle+\operatorname{Ric}\left(\nabla f_{\epsilon}, \nabla f_{\epsilon}\right)+\left|\operatorname{Hess} f_{\epsilon}\right|^{2}\right) \mathrm{dm}_{1} \int_{X_{2}} \phi u^{2} \mathrm{~d} \mathfrak{m}_{2} \\
& +\int_{X_{1}} \psi_{\epsilon}\left|\nabla f_{\epsilon}\right|^{2} \mathrm{dm}_{1} \int_{X_{2}} \phi u \Delta u \mathrm{dm}_{2}+\int_{X_{1}} \psi_{\epsilon}\left(f_{\epsilon} \Delta f_{\epsilon}+2\left|\nabla f_{\epsilon}\right|^{2}\right) \mathrm{d} \mathfrak{m}_{1} \int_{X_{2}} \phi|\nabla u|^{2} \mathrm{~d} \mathfrak{m}_{2} \\
& +\frac{1}{2} \int_{X_{1}} \psi_{\epsilon} f_{\epsilon}^{2} \mathrm{dm}_{1} \int_{X_{2}} \Delta \phi|\nabla u|^{2} \mathrm{dm}_{2} .
\end{aligned}
$$


Here, we used the Bochner formula

$$
\frac{1}{2} \Delta\left|\nabla f_{\epsilon}\right|^{2}=\left\langle\nabla \Delta f_{\epsilon} \nabla f_{\epsilon}\right\rangle+\operatorname{Ric}\left(\nabla f_{\epsilon}, \nabla f_{\epsilon}\right)+\left|\operatorname{Hess} f_{\epsilon}\right|^{2}
$$

and the equation

$$
\int_{X_{2}}(\Delta \phi) u^{2} \mathrm{~d} \mathfrak{m}_{2}=2 \int_{X_{2}} \phi u \Delta u \mathrm{dm}_{2}+2 \int_{X_{2}} \phi|\nabla u|^{2} \mathrm{~d} \mathfrak{m}_{2},
$$

which can be justified by approximating $u$ by $P_{t} u$.

We have

$$
\begin{aligned}
\int_{M} \psi_{\epsilon} \phi\left\langle\nabla \Delta\left(f_{\epsilon} u\right), \nabla\left(f_{\epsilon} u\right)\right\rangle \mathrm{d} \mathfrak{m} \\
=\int_{X_{1}} \psi_{\epsilon}\left\langle\nabla \Delta f_{\epsilon}, \nabla f_{\epsilon}\right\rangle \mathrm{d}_{1} \int_{X_{2}} \phi u^{2} \mathrm{~d} \mathfrak{m}_{2}+\int_{X_{1}} \psi_{\epsilon}\left|\nabla f_{\epsilon}\right|^{2} \mathrm{~d}_{1} \int_{X_{2}} \phi u \Delta u \mathrm{~d} \mathfrak{m}_{2} \\
\quad+\int_{X_{1}} \psi_{\epsilon} f_{\epsilon} \Delta f_{\epsilon} \mathrm{d}_{1} \int_{X_{2}} \phi|\nabla u|^{2} \mathrm{~d} \mathfrak{m}_{2}+\int_{X_{1}} \psi_{\epsilon} f_{\epsilon}^{2} \mathrm{dm}_{1} \int_{X_{2}} \phi\langle\nabla \Delta u, \nabla u\rangle \mathrm{d}_{2} .
\end{aligned}
$$

We have

$$
\begin{aligned}
& K \int_{M} \psi_{\epsilon} \phi\left|\nabla\left(f_{\epsilon} u\right)\right|^{2} \mathrm{~d} \mathfrak{m} \\
& \quad=K \int_{X_{1}} \psi_{\epsilon}\left|\nabla f_{\epsilon}\right|^{2} \mathrm{~d} \mathfrak{m}_{1} \int_{X_{2}} \phi u^{2} \mathrm{~d} \mathfrak{m}_{2}+K \int_{X_{1}} \psi_{\epsilon} f_{\epsilon}^{2} \mathrm{dm}_{1} \int_{X_{2}} \phi|\nabla u|^{2} \mathrm{~d} \mathfrak{m}_{2} .
\end{aligned}
$$

We have

$$
\begin{aligned}
\frac{1}{N} \int_{M} \psi_{\epsilon} \phi\left(\Delta\left(f_{\epsilon} u\right)\right)^{2} \mathrm{~d} \mathfrak{m} \\
=\frac{1}{N} \int_{X_{1}} \psi_{\epsilon}\left(\Delta f_{\epsilon}\right)^{2} \mathrm{~d} \mathfrak{m}_{1} \int_{X_{2}} \phi u^{2} \mathrm{~d} \mathfrak{m}_{2}+\frac{2}{N} \int_{X_{1}} \psi_{\epsilon} f_{\epsilon} \Delta f_{\epsilon} \mathrm{d}_{\mathfrak{m}_{1}} \int_{X_{2}} \phi u \Delta u \mathrm{dm}_{2} \\
\quad+\frac{1}{N} \int_{X_{1}} \psi_{\epsilon} f_{\epsilon}^{2} \mathrm{dm}_{1} \int_{X_{2}} \phi(\Delta u)^{2} \mathrm{dm}_{2} .
\end{aligned}
$$

Take $\widetilde{K}>0$ such that $\operatorname{Ric}_{X_{1}} \leq \widetilde{K} g_{X_{1}}$. Then, we get

$$
\begin{aligned}
& \frac{1}{2} \int_{X_{1}} \psi_{\epsilon} f_{\epsilon}^{2} \mathrm{~d}_{1} \int_{X_{2}} \Delta \phi|\nabla u|^{2} \mathrm{dm}_{2} \\
& \geq-\int_{X_{1}} \psi_{\epsilon}\left((\widetilde{K}-K)\left|\nabla f_{\epsilon}\right|^{2}+\left|\operatorname{Hess} f_{\epsilon}-\frac{\Delta f_{\epsilon}}{n} g_{X_{1}}\right|^{2}\right) \mathrm{d} \mathfrak{m}_{1} \int_{X_{2}} \phi u^{2} \mathrm{~d} \mathfrak{m}_{2} \\
&-2 \int_{X_{1}} \psi_{\epsilon}\left|\nabla f_{\epsilon}\right|^{2} \mathrm{~d} \mathfrak{m}_{1} \int_{X_{2}} \phi|\nabla u|^{2} \mathrm{~d} \mathfrak{m}_{2} \\
&-\frac{1}{N n(N-n)} \int_{M} \psi_{\epsilon} \phi\left((N-n)\left(\Delta f_{\epsilon}\right) u-n f_{\epsilon} \Delta u\right)^{2} \mathrm{~d} \mathfrak{m} \\
&+\int_{X_{1}} \psi_{\epsilon} f_{\epsilon}^{2} \mathrm{dm}_{1} \int_{X_{2}} \phi\left(\langle\nabla \Delta u, \nabla u\rangle+K|\nabla u|^{2}+\frac{1}{N-n}(\Delta u)^{2}\right) \mathrm{d} \mathfrak{m}_{2}
\end{aligned}
$$

by (3.2)-(3.6). Since we have

$$
0<\int_{X_{1}} \psi_{\epsilon} \mathrm{d} \mathfrak{m}_{1} \leq \int_{X_{1}} \psi_{\epsilon} f_{\epsilon}^{2} \mathrm{dm}_{1} \leq\left(1+\epsilon^{2}\right)^{2} \int_{X_{1}} \psi_{\epsilon} \mathrm{d} \mathfrak{m}_{1}
$$


and

$$
\begin{gathered}
\left\|\sqrt{\psi_{\epsilon} \phi}\left((N-n)\left(\Delta f_{\epsilon}\right) u-n f_{\epsilon} \Delta u-2 n(N-n) u+n \Delta u\right)\right\|_{L^{2}} \\
\leq C \epsilon\left(\|\sqrt{\phi} u\|_{L^{2}}+\|\sqrt{\phi} \Delta u\|_{L^{2}}\right)\left(\int_{X_{1}} \psi_{\epsilon} \mathrm{dm}_{1}\right)^{1 / 2}
\end{gathered}
$$

we get the claim by (3.1).

Letting $\epsilon \rightarrow 0$ in Claim 3.17, we get the proposition.

Let us complete the proof of Proposition 3.10. Since we have already showed Corollary 3.12, we only need to check the $\operatorname{BL}(K, N-n)$ condition for $\left(X_{2}, d_{2}, \mathfrak{m}_{2}\right)$. The proof of the following proposition has been inspired by the proof of [24, Theorem 1.2].

Proposition 3.18. In addition to Assumption 3.9, we assume that $n$ is an integer with $N-n \geq 1$ and that $\left(X_{1}, d_{1}, \mathfrak{m}_{1}\right)$ is an $n$-dimensional closed Riemannian manifold with the Riemannian distance and $\mathfrak{m}_{1}=\underline{\mathcal{H}}^{n}$. Then, the metric measure space $\left(X_{2}, d_{2}, \mathfrak{m}_{2}\right)$ satisfies the $\operatorname{BL}(K, N-n)$ condition.

Proof. Similarly to the proof of the assertion that the $\mathrm{BE}(K, N)$ condition implies the $\mathrm{BL}(K, N)$ condition [14, Proposition 4.9], we have the following claim:

Claim 3.19. For any $u \in \mathcal{D}\left(\Delta_{X_{2}}\right)$ and $t>0$, we have

$$
\left|\nabla P_{t} u\right|^{2}+\frac{1-\mathrm{e}^{-2 K t}}{K(N-n)}\left(\left(\Delta P_{t} u\right)^{2}-\frac{2 n}{N} P_{t}\left((\Delta u-2(N-n) u)^{2}\right)\right) \leq \mathrm{e}^{-2 K t} P_{t}\left(|\nabla u|^{2}\right)
$$

$\mathfrak{m}_{2}$-a.e. in $X_{2}$.

Proof. Take arbitrary $\phi \in L^{\infty}\left(X_{2}\right)$ with $\phi \geq 0$. Define $h:[0, t] \rightarrow \mathbb{R}$ by

$$
h(s):=\mathrm{e}^{-2 K s} \int_{X_{2}} P_{s} \phi\left|\nabla P_{t-s} u\right|^{2} \mathrm{~d} \mathfrak{m}_{2} .
$$

Then, for each $0<s<t$, we have

$$
\begin{aligned}
\frac{\partial}{\partial s} h(s)= & -2 K \mathrm{e}^{-2 K s} \int_{X_{2}} P_{s} \phi\left|\nabla P_{t-s} u\right|^{2} \mathrm{dm}_{2} \\
& +\mathrm{e}^{-2 K s} \int_{X_{2}} \Delta P_{s} \phi\left|\nabla P_{t-s} u\right|^{2} \mathrm{dmm}_{2}-2 \mathrm{e}^{-2 K s} \int_{X_{2}} P_{s} \phi\left\langle\nabla \Delta P_{t-s} u, \nabla P_{t-s} u\right\rangle \mathrm{dm}_{2} \\
\geq & 2 \frac{\mathrm{e}^{-2 K s}}{N-n}\left(\int_{X_{2}} P_{s} \phi\left(\Delta P_{t-s} u\right)^{2} \mathrm{~d} \mathfrak{m}_{2}-\frac{2 n}{N} \int_{X_{2}} P_{s} \phi\left(\Delta P_{t-s} u-2(N-n) P_{t-s} u\right)^{2} \mathrm{~d} \mathfrak{m}_{2}\right) \\
\geq & 2 \frac{\mathrm{e}^{-2 K s}}{N-n}\left(\int_{X_{2}} \phi\left(\Delta P_{t} u\right)^{2} \mathrm{~d} \mathfrak{m}_{2}-\frac{2 n}{N} \int_{X_{2}} \phi P_{t}\left((\Delta u-2(N-n) u)^{2}\right) \mathrm{dm}_{2}\right) .
\end{aligned}
$$

Here, we used

$$
\begin{aligned}
& \frac{\partial}{\partial s} P_{s} \phi=\Delta P_{s} \phi, \\
& \frac{\partial}{\partial s} P_{t-s} u=-\Delta P_{t-s} u
\end{aligned}
$$


in $W^{1,2},\left|\nabla P_{t-s} u\right|^{2} \leq \mathrm{e}^{-2 K(t-s)} P_{t-s}\left(|\nabla u|^{2}\right)$ (by the $\mathrm{BL}(K, N)$ condition), Proposition 3.16 and the Jensen inequality

$$
\begin{aligned}
& \left(\Delta P_{t} u\right)^{2} \leq P_{s}\left(\left(\Delta P_{t-s} u\right)^{2}\right) \\
& \left(\Delta P_{t-s} u-2(N-n) P_{t-s} u\right)^{2} \leq P_{t-s}\left((\Delta u-2(N-n) u)^{2}\right)
\end{aligned}
$$

$\mathfrak{m}_{2}$-a.e. in $X_{2}$. Combining this and

$$
\begin{aligned}
& \lim _{s \rightarrow 0} h(s)=h(0)=\int_{X_{2}} \phi\left|\nabla P_{t} u\right|^{2} \mathrm{dm}_{2}, \\
& \lim _{s \rightarrow t} h(s)=h(t)=\mathrm{e}^{-2 K t} \int_{X_{2}} \phi P_{t}|\nabla u|^{2} \mathrm{dm}_{2},
\end{aligned}
$$

we get

$$
\begin{aligned}
\int_{X_{2}} \phi & \left(\mathrm{e}^{-2 K t} P_{t}|\nabla u|^{2}-\left|\nabla P_{t} u\right|^{2}\right) \mathrm{d} \mathfrak{m}_{2} \\
& \geq \frac{1-\mathrm{e}^{-2 K t}}{K(N-n)}\left(\int_{X_{2}} \phi\left(\Delta P_{t} u\right)^{2} \mathrm{~d} \mathfrak{m}_{2}-\frac{2 n}{N} \int_{X_{2}} \phi P_{t}\left((\Delta u-2(N-n) u)^{2}\right) \mathrm{d} \mathfrak{m}_{2}\right) .
\end{aligned}
$$

This implies the claim.

Let us show that $\left(X_{2}, d_{2}, \mathfrak{m}_{2}\right)$ satisfies the $\operatorname{BL}(K, N-n)$ condition. Take $u \in W^{1,2}\left(X_{2}\right)$ and fix $s>0$. Define

$$
v:=\Delta P_{s} u-2(N-n) P_{s} u=P_{s / 2} \Delta P_{s / 2} u-2(N-n) P_{s} u \in \operatorname{TestF}\left(X_{2}\right)
$$

and $v_{x}:=v-v(x)$ for each $x \in X_{2}$. Then, the functions $v_{x}: X_{2} \rightarrow \mathbb{R}$ and

$$
[0, \infty) \times X_{2} \rightarrow \mathbb{R}, \quad(t, y) \mapsto P_{t}\left(v_{x}^{2}\right)(y)
$$

are continuous. Thus, for fixed $\epsilon>0$ and any $x \in X_{2}$, there exists $\delta_{x}, \tau_{x}>0$ such that we have

$$
\left|P_{t}\left(v_{x}^{2}\right)(y)\right|<\epsilon
$$

for any $y \in B_{\delta_{x}}(x)$ and $t \in\left(0, \tau_{x}\right)$. Since $X_{2}$ is compact, there exist points $x_{1}, \ldots, x_{k} \in X_{2}$ $\left(k \in \mathbb{Z}_{>0}\right)$ such that

$$
X_{2}=\bigcup_{i} B_{\delta_{x_{i}}}\left(x_{i}\right)
$$

Put $\tau:=\min \tau_{x_{i}}$. Define

$$
\tilde{v}_{i}:=P_{s} u-\left(P_{s} u\right)\left(x_{i}\right)+\frac{1}{2(N-n)} \Delta\left(P_{s} u\right)\left(x_{i}\right) .
$$

Then, we have $\tilde{v}_{i} \in \mathcal{D}\left(\Delta_{X_{2}}\right)$ and

$$
\Delta \tilde{v}_{i}-2(N-n) \tilde{v}_{i}=\Delta P_{s} u-2(N-n) P_{s} u+2(N-n)\left(P_{s} u\right)\left(x_{i}\right)-\Delta\left(P_{s} u\right)\left(x_{i}\right)=v_{x_{i}} .
$$

Applying Claim 3.19 to $\tilde{v}_{i}$, for each $i$ and $t \in(0, \tau)$, we get

$$
\begin{aligned}
\mathrm{e}^{-2 K t} P_{t}\left(\left|\nabla P_{s} u\right|^{2}\right) & \geq\left|\nabla P_{s+t} u\right|^{2}+\frac{1-\mathrm{e}^{-2 K t}}{K(N-n)}\left(\left(\Delta P_{s+t} u\right)^{2}-\frac{2 n}{N} P_{t}\left(v_{x_{i}}^{2}\right)\right) \\
& \geq\left|\nabla P_{s+t} u\right|^{2}+\frac{1-\mathrm{e}^{-2 K t}}{K(N-n)}\left(\left(\Delta P_{s+t} u\right)^{2}-\frac{2 n}{N} \epsilon\right)
\end{aligned}
$$


$\mathfrak{m}_{2}$-a.e. in $B_{\delta_{x_{i}}}\left(x_{i}\right)$. Thus, for each $i$ and $t \in(0, \tau)$, we get

$$
\mathrm{e}^{-2 K t} P_{t}\left(\left|\nabla P_{s} u\right|^{2}\right) \geq\left|\nabla P_{s+t} u\right|^{2}+\frac{1-\mathrm{e}^{-2 K t}}{K(N-n)}\left(\left(\Delta P_{s+t} u\right)^{2}-\frac{2 n}{N} \epsilon\right)
$$

$\mathfrak{m}_{2}$-a.e. in $X_{2}$. Letting $\epsilon \rightarrow 0$, we get

$$
\mathrm{e}^{-2 K t} P_{t}\left(\left|\nabla P_{s} u\right|^{2}\right) \geq\left|\nabla P_{s+t} u\right|^{2}+\frac{1-\mathrm{e}^{-2 K t}}{K(N-n)}\left(\Delta P_{s+t} u\right)^{2}
$$

$\mathfrak{m}_{2}$-a.e. in $X_{2}$. Letting $s \rightarrow 0$, we get the following inequality as the limit in $L^{1}\left(X_{2}\right)$ :

$$
\mathrm{e}^{-2 K t} P_{t}\left(|\nabla u|^{2}\right) \geq\left|\nabla P_{t} u\right|^{2}+\frac{1-\mathrm{e}^{-2 K t}}{K(N-n)}\left(\Delta P_{t} u\right)^{2}
$$

$\mathfrak{m}_{2}$-a.e. in $X_{2}$. This is the $\operatorname{BL}(K, N-n)$ condition.

By Corollary 3.12 and Proposition 3.18, we get Proposition 3.10. By Propositions 3.6 and 3.10, we get Proposition 3.2.

\section{Proof of the Main Theorem}

In this section we complete the proof of our main theorem.

Theorem 4.1. For integers $n \geq 5$ and $2 \leq p<n / 2$ and a positive real number $\epsilon>0$, there exists $\delta=\delta(n, p, \epsilon)>0$ such that the following property holds. Let $(M, g)$ be an $n$-dimensional closed Riemannian manifold with $\operatorname{Ric}_{g} \geq(n-p-1) g$, and assume one of the following:

- $\lambda_{1}\left(\Delta_{C, p}\right) \leq \delta$ and $\lambda_{n-p+1}(g) \leq n-p+\delta$,

- $\lambda_{1}\left(\Delta_{C, n-p}\right) \leq \delta$ and $\lambda_{n-p}(g) \leq n-p+\delta$.

Then, we have

$$
\lambda_{n-p+2}(g) \geq \frac{p(n-p-1)}{p-1}-\epsilon .
$$

By the Lichnerowicz estimate for the first eigenvalue of the Laplacian acting on functions for metric measure spaces satisfying the $\operatorname{RCD}^{*}(n-p-1, p)$ condition [14, Theorem 4.22]:

$$
\lambda_{1} \geq \frac{p(n-p-1)}{p-1}
$$

we get Theorem 4.1 similarly to Theorem 4.2 below. Thus, we only give the proof of Theorem 4.2.

The following theorem is the main result of this article.

Theorem 4.2. For integers $n \geq 5$ and $2 \leq p<n / 2$ and a positive real number $\epsilon>0$, there exists $\delta=\delta(n, p, \epsilon)>0$ such that the following property holds. Let $(M, g)$ be an $n$-dimensional closed Riemannian manifold with $\mathrm{Ric}_{g} \geq(n-p-1) g$ satisfying one of the following:

- $\lambda_{1}\left(\Delta_{C, p}\right) \leq \delta, \lambda_{n-p+1}(g) \leq n-p+\delta$ and $\lambda_{n+1}(g) \leq p(n-p-1) /(p-1)+\delta$,

- $\lambda_{1}\left(\Delta_{C, n-p}\right) \leq \delta, \lambda_{n-p}(g) \leq n-p+\delta$ and $\lambda_{n+1}(g) \leq p(n-p-1) /(p-1)+\delta$.

Then, we have

$$
d_{\mathrm{GH}}\left(M, S^{n-p}(1) \times S^{p}\left(\sqrt{\frac{p-1}{n-p-1}}\right)\right) \leq \epsilon .
$$


Proof. We show the theorem by a contradiction. Suppose that the theorem does not hold. Then, there exists a sequence of $n$-dimensional closed Riemannian manifolds $\left\{\left(M_{i}, g_{i}\right)\right\}_{i=1}^{\infty}$ with $\operatorname{Ric}_{g_{i}} \geq(n-p-1) g_{i}$ that does not converge to $S^{n-p}(1) \times S^{p}(\sqrt{(p-1) /(n-p-1)})$ and that satisfies one of the following:

- $\lim _{i \rightarrow \infty} \lambda_{n-p+1}\left(g_{i}\right)=n-p, \lim _{i \rightarrow \infty} \lambda_{n+1}\left(g_{i}\right)=p(n-p-1) /(p-1)$ and $\lim _{i \rightarrow \infty} \lambda_{1}\left(\Delta_{C, p}, g_{i}\right)=0$,

- $\lim _{i \rightarrow \infty} \lambda_{n-p}\left(g_{i}\right)=n-p, \lim _{i \rightarrow \infty} \lambda_{n+1}\left(g_{i}\right)=p(n-p-1) /(p-1)$ and $\lim _{i \rightarrow \infty} \lambda_{1}\left(\Delta_{C, n-p}, g_{i}\right)=0$.

Taking a subsequence, we have that Assumption 3.1 holds by Propositions 2.18 and 2.19, the Gromov compactness theorem (see also [27, Theorem 11.1.10], [1, Theorem 4.54]) and [12, Theorems 1.6 and 1.10]. Then, there exists a Borel measure $\mathfrak{m}_{X}$ on $\mathrm{X}$ such that $\mathfrak{m}=\underline{\mathcal{H}}^{n-p} \times \mathfrak{m}_{X}$ holds and $\left(X, \mathfrak{m}_{X}\right)$ satisfies the $\operatorname{RCD}^{*}(n-p-1, p)$ condition by Proposition 3.2. By the spectral convergence theorem [13, Theorem 7.9] and Theorem 4.1, we have

$$
\lambda_{n-p+2}\left(S^{n-p}(1) \times X, \mathfrak{m}\right)=\cdots=\lambda_{n+1}\left(S^{n-p}(1) \times X, \mathfrak{m}\right)=\frac{p(n-p-1)}{p-1} .
$$

Since the spectrum of the Laplacian on $\left(S^{n-p}(1) \times X, \mathfrak{m}\right)$ coincides with

$$
\left\{\lambda_{i}\left(S^{n-p}(1), \underline{\mathcal{H}}^{n-p}\right)+\lambda_{j}\left(X, \mathfrak{m}_{X}\right): i, j \in \mathbb{Z}_{\geq 0}\right\}
$$

and $\lambda_{n-p+2}\left(S^{n-p}(1), \underline{\mathcal{H}}^{n-p}\right)=2(n-p+1)>p(n-p-1) /(p-1)$, we get that

$$
\lambda_{1}\left(X, \mathfrak{m}_{X}\right)=\cdots=\lambda_{p}\left(X, \mathfrak{m}_{X}\right)=\frac{p(n-p-1)}{p-1} .
$$

By the Obata Rigidity theorem for metric measure spaces satisfying the $\mathrm{RCD}^{*}$ condition [25, Theorem 1.4] with scaling, we have that $\left(X, \mathfrak{m}_{X}\right)$ is isomorphic to either $\left(S^{p}\left(r_{n, p}\right), \underline{\mathcal{H}}^{p}\right)$ or $\left(S_{+}^{p}\left(r_{n, p}\right), \underline{\mathcal{H}}^{p}\right)$, where $r_{n, p}:=\sqrt{(p-1) /(n-p-1)}$ and $S_{+}^{p}\left(r_{n, p}\right)$ denotes the $p$-dimensional hemisphere with radius $r_{n, p}$. In particular, $\left\{\left(M_{i}, g_{i}, \underline{\mathcal{H}}^{n}\right)\right\}$ is a non-collapsing sequence. Thus, we get $\left(X, \mathfrak{m}_{X}\right)$ is isomorphic to $\left(S^{p}\left(r_{n, p}\right), \underline{\mathcal{H}}^{p}\right)$ by [12, Theorem 6.2]. This contradicts to the assumption, and so we get the theorem.

\section{Acknowledgements}

I am grateful to Professor Shouhei Honda for helpful discussions. I also thank Professor Dario Trevisan for answering my questions about the regular Lagrangian flow. I wish to thank the referees for careful reading of the paper and making valuable suggestions. This work was supported by RIKEN Special Postdoctoral Researcher Program.

\section{References}

[1] Aino M., Lichnerowicz-Obata estimate, almost parallel $p$-form and almost product manifolds, arXiv:1904.06533.

[2] Ambrosio L., Gigli N., Savaré G., Calculus and heat flow in metric measure spaces and applications to spaces with Ricci bounds from below, Invent. Math. 195 (2014), 289-391, arXiv:1106.2090.

[3] Ambrosio L., Gigli N., Savaré G., Metric measure spaces with Riemannian Ricci curvature bounded from below, Duke Math. J. 163 (2014), 1405-1490, arXiv:1109.0222.

[4] Ambrosio L., Gigli N., Savaré G., Bakry-Émery curvature-dimension condition and Riemannian Ricci curvature bounds, Ann. Probab. 43 (2015), 339-404, arXiv:1209.5786.

[5] Ambrosio L., Honda S., New stability results for sequences of metric measure spaces with uniform Ricci bounds from below, in Measure Theory in Non-Smooth Spaces, Partial Differ. Equ. Meas. Theory, De Gruyter Open, Warsaw, 2017, 1-51, arXiv:1605.07908. 
[6] Ambrosio L., Trevisan D., Well-posedness of Lagrangian flows and continuity equations in metric measure spaces, Anal. PDE 7 (2014), 1179-1234, arXiv:1402.4788.

[7] Ambrosio L., Trevisan D., Lecture notes on the DiPerna-Lions theory in abstract measure spaces, Ann. Fac. Sci. Toulouse Math. 26 (2017), 729-766, arXiv:1505.05292.

[8] Aubry E., Pincement sur le spectre et le volume en courbure de Ricci positive, Ann. Sci. École Norm. Sup. (4) 38 (2005), 387-405, arXiv:math.DG/0505408.

[9] Burago D., Burago Y., Ivanov S., A course in metric geometry, Graduate Studies in Mathematics, Vol. 33, Amer. Math. Soc., Providence, RI, 2001.

[10] Cavalletti F., Milman E., The globalization theorem for the curvature dimension condition, arXiv:1612.07623.

[11] Cheeger J., Differentiability of Lipschitz functions on metric measure spaces, Geom. Funct. Anal. 9 (1999), $428-517$.

[12] Cheeger J., Colding T.H., On the structure of spaces with Ricci curvature bounded below. I, J. Differential Geom. 46 (1997), 406-480.

[13] Cheeger J., Colding T.H., On the structure of spaces with Ricci curvature bounded below. III, J. Differential Geom. 54 (2000), 37-74.

[14] Erbar M., Kuwada K., Sturm K.-T., On the equivalence of the entropic curvature-dimension condition and Bochner's inequality on metric measure spaces, Invent. Math. 201 (2015), 993-1071, arXiv:1303.4382.

[15] Gigli N., Nonsmooth differential geometry - an approach tailored for spaces with Ricci curvature bounded from below, Mem. Amer. Math. Soc. 251 (2018), v+161, arXiv:1407.0809.

[16] Gigli N., Pasqualetto E., Lectures on nonsmooth differential geometry, SISSA Springer Series, Vol. 2, Springer, 2020.

[17] Gigli N., Rigoni C., Recognizing the flat torus among $\operatorname{RCD}^{*}(0, N)$ spaces via the study of the first cohomology group, Calc. Var. Partial Differential Equations 57 (2018), 104, 39 pages, arXiv:1705.04466.

[18] Grosjean J.-F., A new Lichnerowicz-Obata estimate in the presence of a parallel p-form, Manuscripta Math. 107 (2002), 503-520.

[19] Hajłasz P., Koskela P., Sobolev meets Poincaré, C. R. Acad. Sci. Paris Sér. I Math. 320 (1995), 1211-1215.

[20] Hajłasz P., Koskela P., Sobolev met Poincaré, Mem. Amer. Math. Soc. 145 (2000), x+101 pages.

[21] Honda S., Ricci curvature and almost spherical multi-suspension, Tohoku Math. J. 61 (2009), 499-522.

[22] Honda S., Ricci curvature and $L^{p}$-convergence, J. Reine Angew. Math. 705 (2015), 85-154, arXiv:1212.2052.

[23] Jiang R., Li H., Zhang H., Heat kernel bounds on metric measure spaces and some applications, Potential Anal. 44 (2016), 601-627, arXiv:1407.5289.

[24] Ketterer C., Cones over metric measure spaces and the maximal diameter theorem, J. Math. Pures Appl. 103 (2015), 1228-1275, arXiv:1311.1307.

[25] Ketterer C., Obata's rigidity theorem for metric measure spaces, Anal. Geom. Metr. Spaces 3 (2015), 278295, arXiv:1410.5210.

[26] Petersen P., On eigenvalue pinching in positive Ricci curvature, Invent. Math. 138 (1999), 1-21.

[27] Petersen P., Riemannian geometry, 3rd ed., Graduate Texts in Mathematics, Vol. 171, Springer, Cham, 2016.

[28] Rajala T., Local Poincaré inequalities from stable curvature conditions on metric spaces, Calc. Var. Partial Differential Equations 44 (2012), 477-494, arXiv:1107.4842.

[29] Sturm K.-T., Analysis on local Dirichlet spaces. II. Upper Gaussian estimates for the fundamental solutions of parabolic equations, Osaka J. Math. 32 (1995), 275-312.

[30] Sturm K.-T., Analysis on local Dirichlet spaces. III. The parabolic Harnack inequality, J. Math. Pures Appl. 75 (1996), 273-297.

[31] Sturm K.-T., On the geometry of metric measure spaces. I, Acta Math. 196 (2006), 65-131.

[32] Sturm K.-T., On the geometry of metric measure spaces. II, Acta Math. 196 (2006), 133-177. 